\title{
C/EBP $\beta$ deletion in oncogenic Ras skin tumors is a synthetic lethal event
}

Zachary J. Messenger ${ }^{1}$, Jonathan R. Hall ${ }^{1,2,3}$, Dereje D. Jima ${ }^{2,4}$, John S. House $\mathbb{C l}^{2,4}$, Hann W. Tam', Debra A. Tokarz ${ }^{2,5}$ and Robert C. Smart ${ }^{1,2,3}$

\begin{abstract}
Therapeutic targeting of specific genetic changes in cancer has proven to be an effective therapy and the concept of synthetic lethality has emerged. CCAAT/enhancer-binding protein- $\beta$ (C/EBP $\beta$ ), a basic leucine zipper transcription factor, has important roles in cellular processes including differentiation, inflammation, survival, and energy metabolism. Using a genetically engineered mouse model, we report that the deletion C/EBP $\beta$ in pre-existing oncogenic Ha-Ras mouse skin tumors in vivo resulted in rapid tumor regression. Regressing tumors exhibited elevated levels of apoptosis and p53 protein/activity, while adjacent C/EBP $\beta$-deleted skin did not. These results indicate that the deletion of C/EBP $\beta$ de-represses p53 in oncogenic Ras tumors but not in normal wild-type Ras keratinocytes, and that C/EBP $\beta$ is essential for survival of oncogenic Ras tumors. Co-deletion of C/EBP $\beta$ and p53 in oncogenic Ras tumors showed p53 is required for tumor regression and elevated apoptosis. In tumors, loss of a pathway that confers adaptability to a stress phenotype of cancer/tumorigenesis, such as DNA damage, could result in selective tumor cell killing. Our results show that oncogenic Ras tumors display a significant DNA damage/replicative stress phenotype and these tumors have acquired a dependence on C/EBP $\beta$ for their survival. RNAseq data analysis of regressing tumors deleted of C/EBP $\beta$ indicates a novel interface between p53, type-1 interferon response, and death receptor pathways, which function in concert to produce activation of extrinsic apoptosis pathways. In summary, the deletion of C/EBP $\beta$ in oncogenic Ras skin tumors is a synthetic lethal event, making it a promising target for future potential anticancer therapies.
\end{abstract}

\section{Introduction}

Ras proteins are small GTPase membrane-bound signal transducers and the coding genes are one of the most frequently mutated dominant oncogenic drivers in human cancer $^{1-3}$. At least one of the three family members (Ha, N, or Ki-RAS) is mutated in $20-30 \%$ of all human cancers, with some cancers exhibiting high percentages of Ras mutation; e.g., $>90 \%$ of pancreatic cancers contain mutated Ki-RAS ${ }^{1}$. Once mutated, oncogenic Ras signaling promotes proliferation through activation of

\footnotetext{
Correspondence: Jonathan R. Hall (jrhall@ncsu.edu) or

Robert C. Smart (rcsmart@ncsu.edu)

${ }^{1}$ Toxicology Program, Raleigh, NC, USA

${ }^{2}$ Center of Human Health and the Environment, Raleigh, NC, USA

Full list of author information is available at the end of the article.

Edited by G. Melino
}

the RAF-MAPK, PI3K, and RAL-GDS pathways ${ }^{4-9}$. These pathways also downregulate pro-apoptotic genes, leading to conditions that favor expansion while evading cell death ${ }^{10}$.

CCAAT/enhancer binding protein- $\beta$ (C/EBP $\beta)$, a basic leucine zipper transcription factor, has important roles in cellular processes including differentiation, inflammation, survival, and energy metabolism ${ }^{11-13} \cdot C / E B P \beta$ is activated by numerous cytokines ${ }^{14-16}$, as well as by oncogenic Ras, RTKs, and Toll-like receptors ${ }^{17-21}$. C/EBP $\beta$ contributes to cell survival in response to DNA damage, toxicants, or oncogenic stress ${ }^{22-25}$. C/EBP $\beta$ levels are increased in numerous human cancers and often are associated with poor prognoses and invasive growth ${ }^{26-35}$. In numerous cancer cell types, C/EBP $\beta$ has a prosurvival function $^{18,22,24,25,32,36}$.

\section{(c) The Author(s) 2018}

(c) Open Access This article is licensed under a Creative Commons Attribution 4.0 International License, which permits use, sharing, adaptation, distribution and reproduction cc) in any medium or format, as long as you give appropriate credit to the original author(s) and the source, provide a link to the Creative Commons license, and indicate if changes were made. The images or other third party material in this article are included in the article's Creative Commons license, unless indicated otherwise in a credit line to the material. If material is not included in the article's Creative Commons license and your intended use is not permitted by statutory regulation or exceeds the permitted use, you will need to obtain permission directly from the copyright holder. To view a copy of this license, visit http://creativecommons.org/licenses/by/4.0/. 
In order for tumor cells to acquire the hallmark traits of cancer ${ }^{37}$, which includes the evasion of apoptosis, tumor cells must respond to and overcome the cellular stresses associated with tumorigenesis ${ }^{38}$. These stresses are often referred to as the "stress phenotypes of tumorigenesis/cancer" and include DNA damage, DNA replicative stress, mitotic stress, metabolic stress, proteotoxic stress, and oxidative stress ${ }^{38}$. Loss of a pathway(s) that confers adaptability to stress phenotypes of tumorigenesis could result in selective tumor cell killing ${ }^{38}$. These ideas form the conceptual framework of synthetic lethality, where targeting a specific pathway results in death of tumor cells but has no effect in normal cells ${ }^{39-42}$.

Our results demonstrate that deletion of C/EBP $\beta$ in oncogenic Ras-driven skin tumors is a synthetic lethal event. C/EBP $\beta$-depleted tumors displayed activation of a type-1 interferon (IFN) response and de-repression p53 activity to induce death receptor/tumor necrosis factor receptor (TNFR)-mediated apoptosis and tumor regression.

\section{Materials and methods}

\section{Animal care, treatment/doses, tumor measuring}

All animal husbandry, care, and experimentation was conducted per National Institute of Health $(\mathrm{NIH})$ guidelines and approved by the North Carolina State University (NCSU) Institutional Animal Care and Use Committee (IACUC). All mice were backcrossed onto a B6.129 background for at least five generations. K14-CreER ${ }^{\text {tam }}$ mice (from Jackson Laboratory $\mathrm{Tg}$ (KRT14-cre/ERT) $20 \mathrm{EFu} / \mathrm{J})^{43}$ were crossed with $\mathrm{C} / \mathrm{EBP} \beta^{\text {flox } / \text { lox }}$ mice $^{44}$ and p53 ${ }^{\text {flox/flox }}$ mice (from NCI Mouse Repository FVB.129$\left.\operatorname{Trp} 53^{\mathrm{tm} 1 \mathrm{Brn}}\right)^{45}$ to obtain the following genotypes, which have been maintained on a B6.129 hybrid background: $\mathrm{K}_{14-C r e E R^{\mathrm{tam}}}$ (Cre), K14-CreER ${ }^{\text {tam }}$ C/EBP $\beta^{\text {flox/flox }}$ (IKO 3$), \quad K 14-C r e E R^{\text {tam;}} ; p_{53} 3^{\text {flox/flox }}$ (IKOp53), and K14$\mathrm{CreER}^{\text {tam }} ; \mathrm{C} / \mathrm{EBP} \beta^{\text {flox/flox }} ; \mathrm{p} 53^{\text {flox/flox }}$ (DIKO). To induce tumors, mice aged 8-12 weeks had dorsal hair clipped with electric clippers and were given a single topical dose of $200 \mathrm{nmol}$ 7,12-dimethylbenz[a]anthracene (DMBA) $(0.2 \mathrm{ml})$ (Sigma, D3254, St. Louis, MO, USA) in acetone followed 1 week later by thrice weekly dosing of $5 \mathrm{nmol}$ 12-O-tetradecanoylphorbol-13-acetate (TPA) (0.2 ml) (Cayman Chemical, 10008014, Ann Arbor, MI, USA) in acetone for the entirety of tumor studies. Activation of the K14-CreER ${ }^{\text {tam }}$ was accomplished via dosing $2.5 \mathrm{mg}$ (6.73 $\mu$ mol) of tamoxifen (Sigma, T5648, St. Louis MO, USA) dissolved in corn oil with $5 \%$ ethanol intraperitoneal (i.p.) injected daily $(0.25 \mathrm{ml}), 5$ days/week for 2 weeks. Tumor numbers were tabulated weekly with tumor volume being calculated by measuring height, width, and length of tumors (height $\times$ width $\times$ length). In vivo 5-Bromo2'deoxyuridine (BrdU) labeling was carried out as previously described ${ }^{22}$ by i.p. injecting BrdU (Sigma, B5002,
St. Louis, MO, USA) in phosphate-buffered saline (PBS, $\mathrm{pH}$ 7.4) $1 \mathrm{~h}$ before killing at a dose of $100 \mathrm{mg}$ BrdU per $\mathrm{kg}$ body mass (typically $\sim 0.3 \mathrm{ml}$ ).

\section{DNA sequencing}

Whole tumor DNA was collected and Sanger sequencing was performed by the North Carolina State University Genomic Sciences Laboratory (Raleigh, NC, USA). The 61st codon of Ha-Ras was amplified using forward primer 5'-ACTCCTACCGGAAACAGGT-3' and reverse primer: 5'-GAGGACATCCATCAGTACAG-3', and sequenced using the reverse primer.

\section{Preparation of epidermal lysates for SDS-polyacrylamide gel electrophoresis}

Mice were killed by cervical dislocation and clipped dorsal skin was removed. To remove epidermis, the

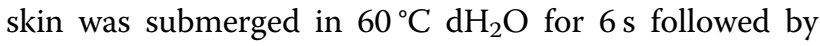
$10 \mathrm{~s}$ in ice water, the skin was dried and the epidermis was scraped off of the dermis and placed in RIPA buffer (1\% NP-40, 0.5\% sodium deoxycholate, $0.1 \%$ SDS, $1 \mathrm{mM}$ dithiothreitol, $1 \mathrm{mM}$ sodium orthovanadate, $1 \mathrm{mM} \mathrm{4-(2-}$ Aminoethyl)benzenesulfonyl fluoride hydrochloride (AEBSF), and $1 \times$ protease inhibitor cocktail (Roche, Indianapolis, IN, USA) in PBS. Collected samples were sonicated on ice and centrifuged at $14,000 \times g$ for $10 \mathrm{~min}$. Equal amounts of protein were resolved via SDSpolyacrylamide gel electrophoresis, transferred to polyvinylidene difluoride membrane and probed using the following antibodies: C/EBP $\beta$ (Santa Cruz, sc-150, 1:5000, Dallas, TX, USA), $\alpha$-Tubulin (Santa Cruz, sc-8035, 1:8000, Dallas, TX, USA), and p53 (Cell Signaling, 2524, 1:5000, Danvers, MA, USA).

\section{Quantification of apoptosis and inflammatory cell infiltration}

Shaved mouse dorsal skin was fixed in 10\% neutral buffered formalin for $24 \mathrm{~h}$, changed to $70 \%$ ethanol, and embedded in paraffin, or tissues were fixed in PAX gene tissue fixative (PreAnalytiX, 765312, Hombrechtikon, Switzerland) for $24 \mathrm{~h}$, changed to PAXgene stabilizer (PreAnalytiX, 765512, Hombrechtikon, Switzerland), and embedded in paraffin. Tissue sections $(5 \mu \mathrm{M})$ were stained with hematoxylin and eosin. Apoptotic cells were scored as positive if they met all three following criteria: (1) dark pyknotic nuclei, (2) cytoplasmic eosinophilia, and (3) detachment from adjacent cells $^{18,22,23}$. Apoptotic positive cells were expressed as positive cells per $\mathrm{mm}^{2}$ area of parenchyna. Area was measured using ImageJ software $^{46}$. Apoptosis in the normal epidermis adjacent to tumors was scored in the interfollicular epidermis as previously described above and expressed as positive cells per $\mathrm{cm}$ skin. Apoptosis was confirmed with cleaved caspase 3 (Cell Signaling, 9661, 1:1000, Danvers, MA, USA) 
immunohistochemical (IHC) and Terminal deoxynucleotidyl transferase dUTP nick end labeling (TUNEL) (Promega, G3250, Madison, WI, USA) staining using UVB-treated mouse skin as a positive control and untreated mouse skin used as negative control. Inflammation was scored based on infiltrating neutrophils and mononuclear leukocytes, which were scored separately for intratumoral and extratumoral skin using a scoring system adapted from a method previously described ${ }^{47}$ on a scale of $0-3$, where 0 : none present, 1 : few present, 2: moderate occurrence, and 3: abundant occurrence. Intratumoral included the epidermal components of the papillomas with the subjacent dermis and subcutis. All slides were scored by $\mathrm{D}$. Tokarz (board certified veterinary pathologist from CHHE Comparative Pathology Core), who was blinded as to treatment group, with the exception of slides labeled as controls. Slides were scored in a random order.

\section{Tumor histological analysis}

Using hematoxylin and eosin (H\&E)-stained $5 \mu \mathrm{m} \mathrm{sec}-$ tions, skin lesions were identified and scored by a veterinary pathologist and scored as follows: (1) Papilloma: a discrete mass $>1 \mathrm{~mm}$ in depth that displayed hyperplasia with mild dysplasia. (2) Carcinoma in situ: a discrete raised hyperplasia that is $>1 \mathrm{~mm}$ in depth with expanded dermis. The dysplasia includes the loss of transepidermal differentiation, increased in mitotic index, and increased in nuclear to cytoplasm ratio. (3) Microinvasive squamous cell carcinoma: in addition to the criteria in carcinoma in situ, the lesion has increase depth in dermis with expansion through basement membrane. (4) Squamous cell carcinoma: squamous cell carcinoma that touches or penetrates the muscle layer. Categories (3) and (4) are considered as malignant skin tumors.

\section{Immunohistochemical staining}

Clipped mouse dorsal skin was fixed in $10 \%$ neutral buffered formalin for $24 \mathrm{~h}$, changed to $70 \%$ ethanol, and embedded in paraffin, or tissues were fixed in PAXgene tissue fixative for $24 \mathrm{~h}$, changed to PAXgene stabilizer, and embedded in paraffin. Tissue sections $(5 \mu \mathrm{m})$ were deparaffinized, peroxidases were inactivated with $3 \%$ $\mathrm{H}_{2} \mathrm{O}_{2}$, and subjected to antigen retrieval using a 2100 retriever (Aptum, Southampton, UK) with citrate buffer (pH 6). Next, sections were treated with $3 \% \mathrm{H}_{2} \mathrm{O}_{2}$ once more before being blocked with normal goat serum (or normal horse serum) before incubation overnight at $4{ }^{\circ} \mathrm{C}$ with one of the following antibodies: C/EBP $\beta$ (Santa Cruz, sc-150, 1:4000, Dallas, TX, USA with C/EBP $\beta^{-1-}$ mouse skin used as negative control and wild-type mouse skin used as positive control, p53 (Cell Signaling, 2524, 1:1000, Danvers, MA, USA) with $\mathrm{p} 53^{-1-}$ mouse skin used as negative control and UVB-treated mouse skin used as positive control, Keratin 5 (Covance, PRB-160P, 1:2000, Princeton, NJ, USA) with wild-type mouse skin used as positive control, Keratin 10 (Covance, PRB159P, 1:2000, Princeton, NJ, USA) with wild-type mouse skin used as positive control, Ki67 (Bethyl Labs, IHC-00375, 1:500, Montgomery, TX, USA) with wild-type mouse skin used as positive control, $\gamma \mathrm{H} 2 \mathrm{AX}$ (Bethyl Labs, A300-081A, 1:2000, Montgomery, TX, USA) and phospho-p53 (Ser15) (murine serine 18 (S18)) (Cell Signaling, 9284, 1:1000, Danvers, MA, USA) with skin from a gamma radiation dosed mouse as a positive control and wild-type mouse skin as a negative control, and cleaved caspase 8 using wild-type mouse skin as a negative control. (Novus Biologicals, 56116, 1:2000, Littleton, CO). Staining was visualized using species appropriate secondary antibodies from Vectastain Elite ABC kits (Vector Labs, mouse:PK6102 rabbit:PK-6101, Burlingame CA, USA) and DAB Peroxidase Substrate Kit (Vector Labs, SK-4100, Burlingame, CA, USA). Sections were counterstained with hematoxylin and quantification was calculated as positive cells per $\mathrm{mm}^{2}$ area of parenchyma. Area was measured using ImageJ software ${ }^{46}$. Positively stained cells in the normal epidermis adjacent to tumors were scored in the interfollicular epidermis and expressed as positive cells per $\mathrm{cm}$ skin. Immunohistochemical staining for BrdU was carried out as previously described ${ }^{22}$ by deparaffinizing 5 $\mu \mathrm{m}$ sections and incubating in the following, $2 \mathrm{M} \mathrm{HCl}$ for $30 \mathrm{~min}$ at $37^{\circ} \mathrm{C}$, boric acid-borate buffer for $3 \mathrm{~min}$ at room temperature, $0.05 \mathrm{M}$ Tris- $\mathrm{HCl}\left(\mathrm{pH} 7.8\right.$ in $0.1 \% \mathrm{CaCl}_{2}$ ) with $0.01 \%$ trypsin for $3 \mathrm{~min}$ at $37^{\circ} \mathrm{C}$, and $3 \% \mathrm{H}_{2} \mathrm{O}_{2}$ for $10 \mathrm{~min}$ at room temperature. Sections were blocked in normal horse serum for $30 \mathrm{~min}$ and then incubated for $1 \mathrm{~h}$ at room temperature with anti-BrdU IgG primary antibody (BD Biosciences, 69138, 1:100, San Jose, CA, USA). Staining was visualized and quantified as described above. All IHC staining was performed with listed controls as well as no primary antibody controls. All controls behaved as expected. Immunohistochemical analysis for Mouse CD4 (eBioscience, 14-9766, 1:25, Waltham, MA, USA), Mouse CD8a (eBioscience, 14-0808,1:100, Waltham, MA, USA), and Mouse F4/80 (AbD Serotec, Cat \# MCA497RT, $1: 25$, Hercules, CA, USA) was performed by the Animal Histopathology and Lab Medicine Core located at the University of North Carolina School of Medicine (Chapel Hill, NC, USA).

\section{RNA isolation and next-generation sequencing}

Total RNA was extracted from whole tumors collected from Cre and IKO $\beta$ mice following cessation of tamoxifen treatment as described above (week 21 of TPA) and were homogenized in Qiazol (Qiagen, 79306, Hilden, Germany). RNA extraction was carried out using the QuickRNA MiniPrep Kit (Zymo Research, 11-328, Irvine, CA, USA). Total RNA samples were submitted to the North 
Carolina State University Genomic Sciences Laboratory for Illumina RNA library construction and sequencing. Sample integrity and concentration were evaluated using an RNA 6000 Nano Chip on the Agilent Bioanalyzer 2100 (Agilent, Santa Clara, CA, USA). Purification of messenger RNA (mRNA) was performed using the oligo-dT beads provided in the NEBNext Poly(A) mRNA Magnetic Isolation Module (New England Biolabs, Ipswich, MA, USA). NEBNext Ultra Directional RNA Library Prep Kit (New England Biolabs, Ipswich, MA, USA) and NEBNext Multiplex Oligos for Illumina (New England Biolabs, Ipswich, MA, USA) were used to make the cDNA libraries for Illumina sequencing using the manufacturer-specified protocol, which involved chemically fragmenting the mRNA and priming it with random oligos for first-strand cDNA synthesis. Second-strand cDNA synthesis was then carried out with dUTPs to preserve strand orientation information. The cDNA was purified, end repaired, and "a-tailed" for adaptor ligation. Next, the samples were selected for a final library size of $400-550$ bp using sequential AMPure XP bead isolation (Beckman Coulter, Brea, CA, USA). Library enrichment was performed and specific indexes for each sample were added during the protocol-specified PCR amplification. The amplified library fragments were purified and checked for quality and final concentration using an Agilent 2200 Tapestation (Agilent, Santa Clara, CA, USA). The final quantified libraries were sequenced using Illumina's NextSeq 500 DNA sequencer, utilizing a 75 bp paired end kit (Illumina, San Diego, CA, USA), which gave around 200 million reads for the 8 samples (4 samples/group), which works out to $\sim 25$ million reads/sample. The software package Real Time Analysis (RTA) was used to generate raw bcl, or base call files, which were then de-multiplexed by sample into fastq files for data submission.

RNA-Seq data analysis was conducted in consultation with the Bioinformatics Core of the NCSU Center of Human Health and the Environment. The quality of raw sequence data was assessed using FastQC and the first 12 poor-quality bases were trimmed based on the quality matrix from the FastQC, application. The remaining good quality reads were aligned to the mouse reference genome $(\mathrm{mm} 38$ version 87$)$ using $\mathrm{STAR}^{48}$ aligner. For each replicate sample, per-gene counts of uniquely mapped reads were calculated using htseq-count script from the HTSeq python package. Genes with numerous aliases were removed and represented in the data as a single gene. The count matrix was imported and normalized for sequence depth and distortion, and dispersion was estimated using DESeq $2^{49}$ Bioconductor package in the $\mathrm{R}$ statistical computing environment. Differentially expressed genes were identified after applying multiple testing correction using the Benjamini-Hockeberg procedure ${ }^{50}$ (false discovery rate $(\mathrm{FDR})<0.1$ ).

\section{Gene set enrichment analysis}

Gene set enrichment analysis (GSEA) is a computational method that determines whether an a priori defined set of genes shows statistically significant, concordant differences between two biological states utilizing GSEA software ${ }^{51,52}$. Briefly, the genes from differential expression were ranked based on signed fold change $\times-\log 10$ $p$-value. The rank file contains genes with the strongest upregulation (top), strongest downregulation (bottom), and not changing are in the middle. The analysis was preformed using GseaPreranked application in the GSEA software using mouse C2 MSigDB gene set and default settings. Finally, the enriched pathways $(F D R<0.1)$ were plotted using Enrichment Map Visualization in the same software package and plotted the bar plot for the top 40 enriched pathways.

For visualization of specific pathways, normalized counts from DESeq2 ${ }^{49}$ were row scaled (by gene), grouped by average linkage, and heatmaps generated. Data for each ontology along with adjusted $p$-values are provided in Supplemental Tables 3-6. Ontologies were retrieved March 2017 and combined in the following manner from the curated C2.GSEA MSigDB database available at (http://www.software.broadinstitute.org/gsea/ msigdb):

Interferon - ("INTERFERON-GAMMA SIGNALING PATHWAY\%PANTHER PATHWAY\%P00035" plus "INTERFERON-GAMMA SIGNALING\%REACTOME\% R-HSA-877300.1" plus "INTERFERON SIGNALING\% REACTOME\%R-HSA-913531.1" plus "INTERFERON ALPHA BETA SIGNALING\%REACTOME\%R-HSA909733.1" plus 10 additional OAS genes), p53 ("HALLMARK_P53_PATHWAY\%MSIGDB_C2\%HALLMARK_P53_PATHWAY"), TNF -("HALLMARK_TNFA_SIGNALING_VIA_NFKB\%MSIGDB_C2\%HALLMARK_TNFA_SIGNALING_VIA_NFKB“), and Death Receptor - $\quad$ ("BIOCARTA_DEATH_PATHWAY\% MSIGDB_C2\%BIOCARTA_DEATH_PATHWAY" plus "DEATH RECEPTOR SIGNALLING\%REACTOME DATABASE ID RELEASE 59\%73887").

\section{Results}

Spatial and temporal regulation of C/EBP $\beta$ in epidermis and pre-existing oncogenic Ras skin tumors

$\mathrm{C} / \mathrm{EBP} \beta$ is abundantly expressed throughout the epithelial portion (parenchyma) of oncogenic Ras containing mouse skin tumors (squamous papillomas) arising from the DMBA/ TPA protocol (Fig. 1a). To determine whether C/EBP $\beta$ is essential for the survival of oncogenic Ha-Ras skin tumors, we developed a mouse model, K14-CreER ${ }^{\mathrm{tam}} ; \mathrm{C} / \mathrm{EBP} \beta^{\text {flox/flox }}$ (IKO $\beta$ ), in which C/EBP $\beta$ could be conditionally and temporally deleted in the epidermis and in pre-existing oncogenic Ha-Ras skin tumors (Fig. 1b). As shown in Fig. 1c, dosing IKO $\beta$ mice with tamoxifen i.p. $(1 \times /$ day for 5 days/ 


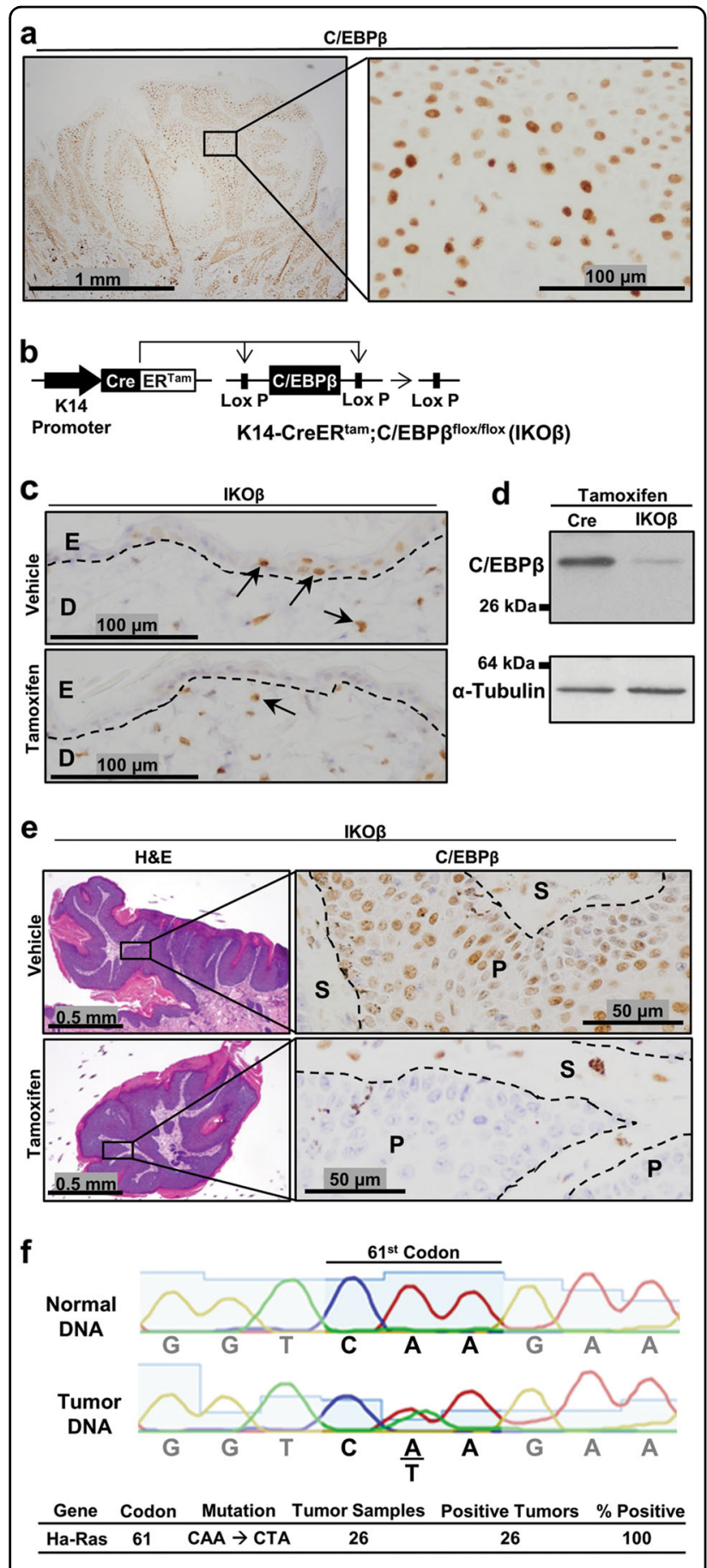

Fig. 1 Spatial and temporal regulation of C/EBP $\beta$ in epidermis and in pre-existing oncogenic Ras skin tumors. a

Immunohistochemical $(\mathrm{HC})$ staining for C/EBP $\beta$ in a DMBATPA-induced mouse squamous papilloma. $\mathbf{b}$ Schematic of the $\mathrm{K} 14-\mathrm{CreER}^{\mathrm{tam}} ; \mathrm{C} / \mathrm{EBP} \beta^{\text {flox }}$ flox (IKOß) transgenic mouse model system. $\mathbf{c} H C$ staining for $C / E B P \beta$ in IKO $\beta$ mouse skin following vehicle or tamoxifen dosing. $D$ dermis, $E$ epidermis. $\mathbf{d}$ Western blot analysis for C/EBP $\beta$ in epidermal lysates from tamoxifen-treated Cre and IKO $\beta$ mice. e H\&E and C/EBP $\beta$ IHC staining in DMBATPA-induced mouse squamous papilloma following vehicle and tamoxifen dosing. $\mathbf{f}$ DNA sequence of the 61st codon of Ha-Ras in mouse tail and DMBATTPA-induced squamous papillomas week for 2 weeks) resulted in loss of $C / E B P \beta$ protein in the epidermis, whereas dermal C/EBP $\beta$ levels were unaffected. Western blot analysis of epidermal lysates confirmed C/EBP $\beta$ deletion (Fig. 1d).

To test whether C/EBP $\beta$ could be deleted in pre-existing skin tumors in this mouse IKO $\beta$ model, we generated skin tumors in IKO $\beta$ mice using a DMBA/TPA tumorigenesis treatment protocol ${ }^{53}$. Following development of oncogenic Ras-driven tumors, mice were then treated with tamoxifen or vehicle i.p. and tumors were collected 2 weeks after the start of tamoxifen treatment. Tamoxifen treatment resulted in the loss of $C / E B P \beta$ protein in the parenchyma portion of the tumor, but not in the stroma (Fig. 1e). DNA sequencing of DMBA/TPA tumors from K14-CreER ${ }^{\text {tam }}$ (Cre) mice confirmed the expected A- $>\mathrm{T}$ mutation in the 61st codon of Ha-Ras (Fig. 1f $)^{54}$. Thus, the IKO $\beta$ mouse is a tractable model to test whether $C / E B P \beta$ is required for survival of oncogenic Ras skin tumors.

\section{Oncogenic Ras skin tumors depend on C/EBP $\beta$ for survival}

Cre and IKO $\beta$ mice were initiated with DMBA and promoted with TPA for 30 weeks. By week 19 of TPA treatment, $100 \%$ of mice from both genotypes developed an average of $\sim 5$ tumors/mouse. At week 19, mice were treated with tamoxifen i.p. $(1 \times /$ day for 5 days/week for 2 weeks). At 10 weeks after the start of tamoxifen treatment, $\sim 80 \%$ of the IKO $\beta$ tumors had completely regressed (Fig. 2a) and the percentage of mice with tumors decreased to $\sim 25 \%$ (Fig. 2b). Using tumor volume at 19 weeks as a standard, tumor volume in Cre mice tripled, whereas it decreased by $98 \%$ in IKO $\beta$ mice after 10 weeks of tamoxifen treatment (Fig. 2c). A representative IKO $\beta$ mouse before and after tamoxifen treatment is shown in Fig. 2d. At the termination of this experiment ( 30 weeks TPA treatment), skin tumors from Cre mice were collected and histopathological analysis showed that $78 \%$ of skin tumors were squamous papillomas and $22 \%$ were squamous cell carcinomas.

To determine whether more progressed skin tumors also regress upon the $C / E B P \beta$ deletion, we conducted another DMBA/TPA tumor study where mice were treated with tamoxifen at 34 weeks of TPA treatment (compared with 19 weeks in the previous experiment). Six weeks after initiation of tamoxifen treatment, $~ 80 \%$ of IKO $\beta$ mouse tumors completely regressed (Fig. 2e) and the percentage of mice with tumors decreased to $50 \%$ (Fig. 2f). This tumor experiment was terminated at 40 weeks, as some tumor bearing mice developed a cachexia-like phenotype. In summary, these results demonstrate that deletion of $\mathrm{C} / \mathrm{EBP} \beta$ in oncogenic Ras tumors results in tumor regression. 

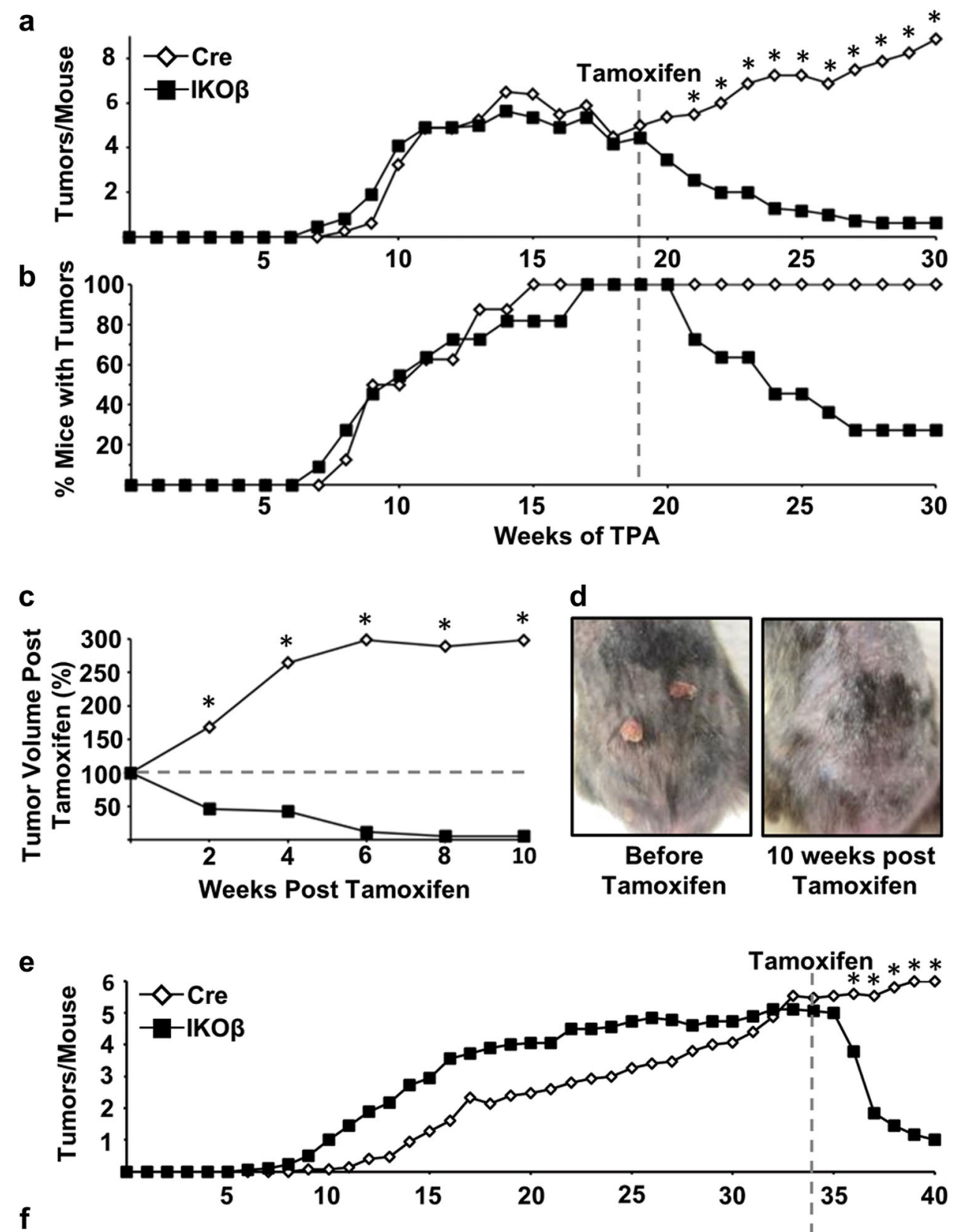

f

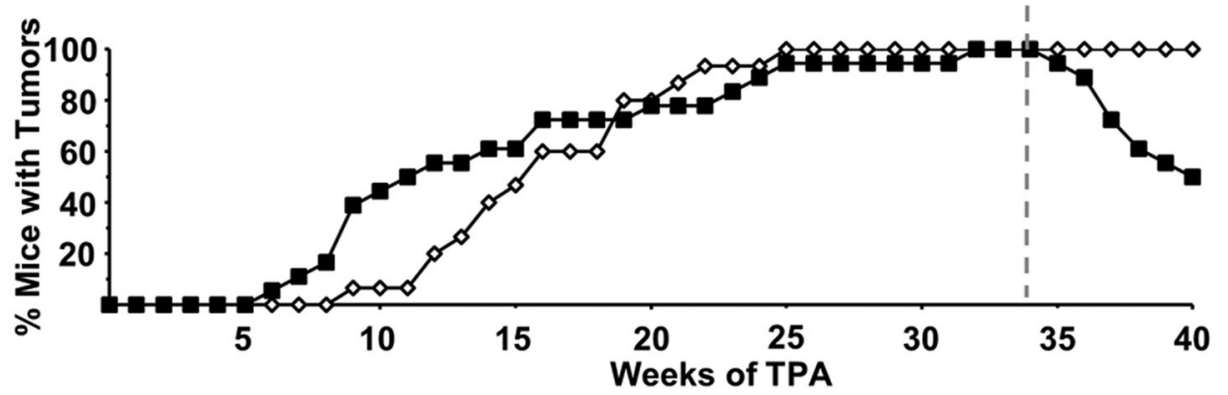

Fig. 2 Oncogenic Ras skin tumors depend on C/EBP $\beta$ for survival. a Tumor multiplicity in Cre and IKO $\beta$ before and after mice were dosed with tamoxifen starting at 19 weeks, $\mathbf{b}$ tumor incidence, and $\mathbf{c}$ tumor volume remaining (Cre mice $n=10$, IKO $\beta$ mice $n=11$ ). $\mathbf{d}$ Representative photographs of skin tumors before and after tamoxifen on the same mouse. e Tumor multiplicity in Cre and IKO $\beta$ mice before and after mice were dosed with tamoxifen starting at 34 weeks, and $\mathbf{f}$ tumor incidence (Cre mice $n=15$, IKO $\beta$ mice $n=18$ ). Data are expressed as means. *indicates significantly different from IKO $\beta$ controls $p<0.05$ via the Student's $t$-test 
Regressing tumors display elevated levels of apoptosis and p53 protein while adjacent C/EBP $\beta$-depleted skin does not

To gain insight into the mechanism of tumor regression, another group of IKO $\beta$ mice were initiated with DMBA, promoted with TPA, and at 19 weeks of TPA treatment, mice were dosed with either tamoxifen or vehicle. Tumors, along with adjacent skin, were collected 2 weeks after the start tamoxifen or vehicle treatment, and PAXgene-fixed paraffin-embedded (PFPE) sections were prepared. The deletion of $\mathrm{C} / \mathrm{EBP} \beta$ in regressing tumors and adjacent skin was confirmed by lack of IHC staining for C/EBP $\beta$ protein (Fig. 3a). Based on the characteristic morphology of apoptotic cells in H\&E-stained sections (Fig. 3b), we observed a $\sim 7$-fold increase in apoptotic cells in the parenchyma of regressing tumors $(\mathrm{C} / \mathrm{EBP} \beta$ deleted) compared with the parenchyma of non-regressing tumors $\left(\mathrm{C} / \mathrm{EBP} \beta^{+/+}\right)$(Fig. 3b). Both TUNEL and cleaved caspase 3 staining produced similar numbers of apoptotic cells as observed in the H\&E sections (data not shown). The increase in apoptosis in the regressing tumors was accompanied by a $\sim 9$-fold increase in p53-positive tumor cells (Fig. 3c). Unlike tumors, there were no significant differences in apoptosis or p53 levels in epidermis adjacent to the tumor when C/EBP $\beta$ was depleted or not (Fig. 3d, e). Moreover, the levels of apoptosis and p53 were low in the adjacent normal epidermis, regardless of $\mathrm{C} / \mathrm{EBP} \beta$ status (Fig. 3d, e). These results demonstrate that the loss of C/EBP $\beta$ leads to increased levels of p53 and apoptotic cell death in oncogenic Ras skin tumor cells but not in adjacent epidermal keratinocytes with wild-type Ras, indicating the loss of $C / E B P \beta$ is a synthetic lethal event in oncogenic Ras tumors. We observed that the increase in p53 protein levels in regressing tumors was not accompanied by increased p53 mRNA levels (Fig. 3f) but was accompanied by increased phosphorylation of S18 of p53 (human serine 15) (Fig. 3g). S18 phosphorylation of p53 is mediated by AtaxiaTelangiesctasia Mutated (ATM)/Ataxia Telangiectasia And Rad3-Related Protein (ATR) in response to DNA damage $e^{5,56}$ and contributes to p53 stabilization and enhances p53-dependent transactivation ${ }^{57}$.

\section{Skin tumors exhibit increased levels of DNA damage, a stress phenotype of tumorigenesis}

Prior work in our lab has demonstrated that the epidermal C/EBP $\beta$-knockout mice treated with DNAdamaging agents display increased levels of p53 and apoptosis compared with similarly treated wild-type mice ${ }^{18,22,23}$. In the current study, the presence of DNA damage and subsequent stress phenotypes in oncogenic Ras skin tumors could provide a mechanism whereby loss of $C / E B P \beta$ results in increased sensitivity to DNA damage, leading to increased p53 levels and apoptosis.
To test for DNA damage, we measured $\gamma \mathrm{H} 2 \mathrm{AX}$, a marker of double-strand breaks ${ }^{58}$ in both tumors and adjacent epidermis. We observed very low numbers of $\gamma \mathrm{H} 2 \mathrm{AX}$ positive cells in the adjacent epidermis of regressing and non-regressing tumors (Fig. 4a). However, $\gamma \mathrm{H} 2 \mathrm{AX}$ IHC positive cells were significantly and similarly increased in both regressing and non-regressing tumors (Fig. 4b). These results indicate that regressing and nonregressing skin tumors display a hallmark tumor stress phenotype involving DNA damage stress ${ }^{38}$. However, only regressing tumors depleted of $\mathrm{C} / \mathrm{EBP} \beta$ display increased p53 (Fig. 3c), phospho-p53(S18) (Fig. 3g), and apoptosis (Fig. 3b), suggesting the activation of p53 in these Ha-Ras regressing tumors occurs via enhancement of pathways that are activated by endogenous DNA damage stress as a result $\mathrm{C} / \mathrm{EBP} \beta$ depletion.

\section{Regressing C/EBP $\beta$-deficient tumors do not display differences in proliferation, senescence, differentiation, or inflammation}

Examination of regressing and non-regressing tumors for BrdU, Ki67, and keratin 5 and 10 showed no difference in proliferation, senescence, or differentiation, respectively (Fig. 4c-e). Further, tumor regression was not accompanied by a significantly different inflammatory response as measured by neutrophil or mononuclear leukocyte infiltration (Fig. 4f-h). IHC staining for CD4, $\mathrm{CD} 8$, and F4/80 antigens indicative of CD4+ T-cells, CD8+ T-cells, and macrophages respectively, revealed no statistically significant differences between regressing and non-regressing tumors as well (Fig. 4i-k). In summary, of the cellular processes evaluated, only increased apoptosis and increased p53 levels are associated with the regressing tumor phenotype.

\section{Development of a mouse model for the inducible conditional co-deletion of C/EBP $\beta$ and p53 in epidermis and in pre-existing oncogenic Ras skin tumors}

To determine whether p53 is required for the observed increase in apoptosis and tumor regression following deletion of $C / E B P \beta$, we developed an inducible conditional C/EBP $\beta-$ p53 double knockout mouse K14$\mathrm{CreER}^{\text {tam }} ; \mathrm{C} / \mathrm{EBP} \beta^{\text {flox/flox }} ; \mathrm{p} 53^{\text {flox/flox }}$ (DIKO) (Fig. 5a) in which $C / E B P \beta$ and p53 could be spatially and temporally co-deleted in the epidermis and in pre-existing oncogenic Ha-Ras skin tumors. Dosing DIKO mice with tamoxifen resulted in the loss of both $C / E B P \beta$ and $p 53$ protein in the epidermis (Fig. 5b, c). To test whether C/EBP $\beta$ and p53 could be co-deleted in pre-existing skin tumors, we generated skin tumors in Cre and DIKO mice using a DMBA/TPA tumorigenesis treatment protocol and treated with tamoxifen. Following tamoxifen treatment, both $\mathrm{C} / \mathrm{EBP} \beta$ and $\mathrm{p} 53$ protein in the epithelial (parenchyma) portion of the DIKO skin tumors were deleted (Fig. 5d). 

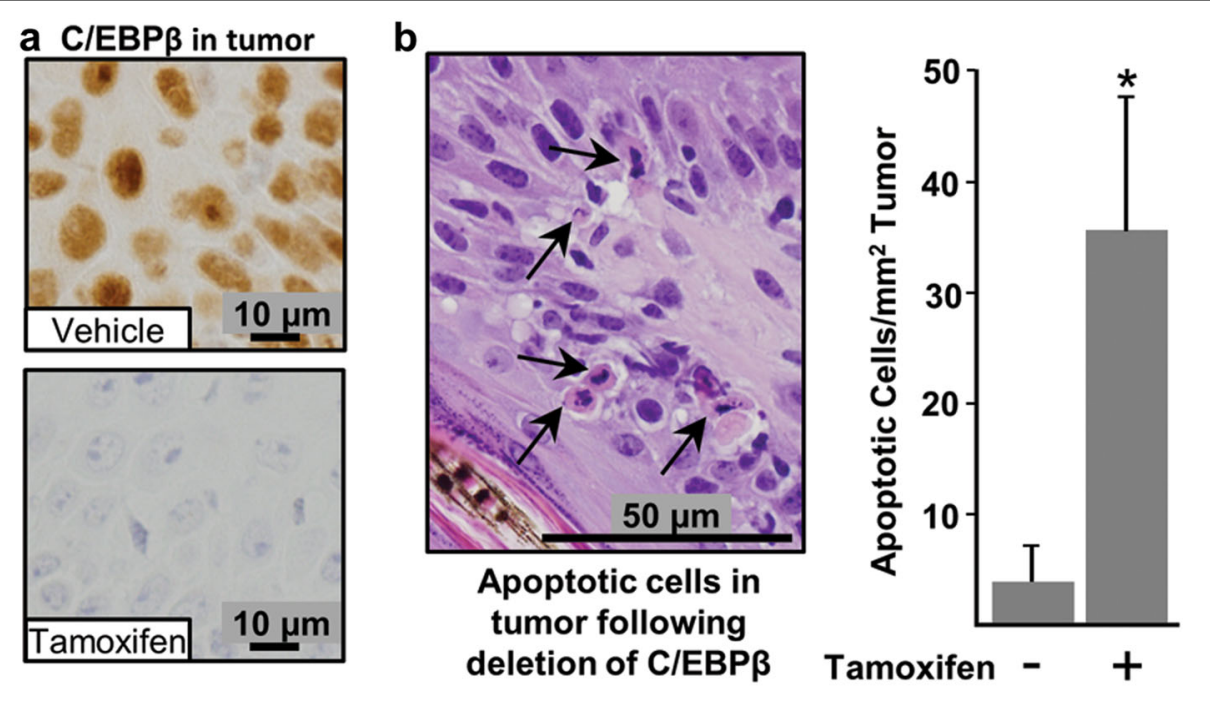

C

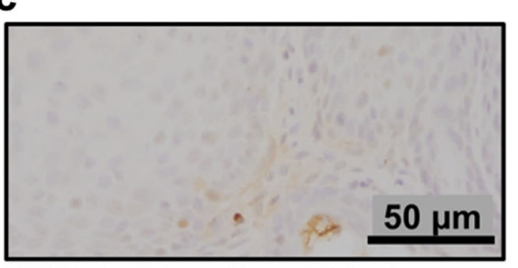

IHC staining for p53 in tumor dosed with Vehicle

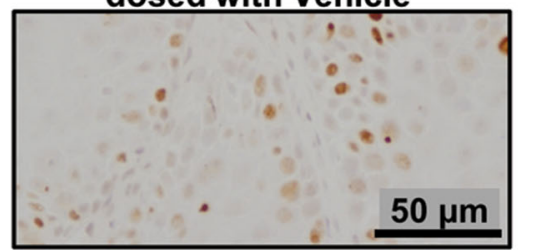

IHC staining for p53 in tumor
dosed with Tamoxifen

e

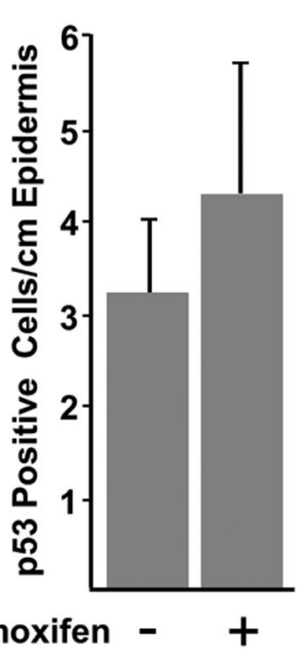

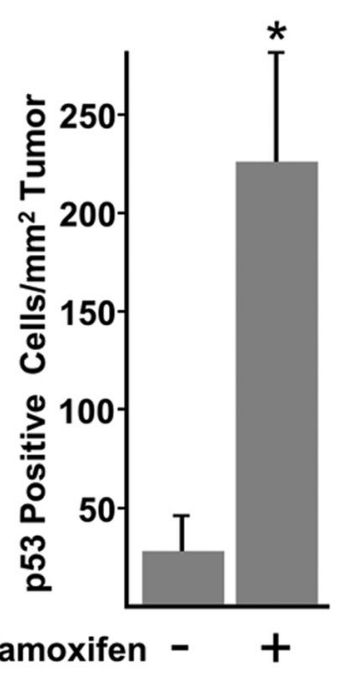
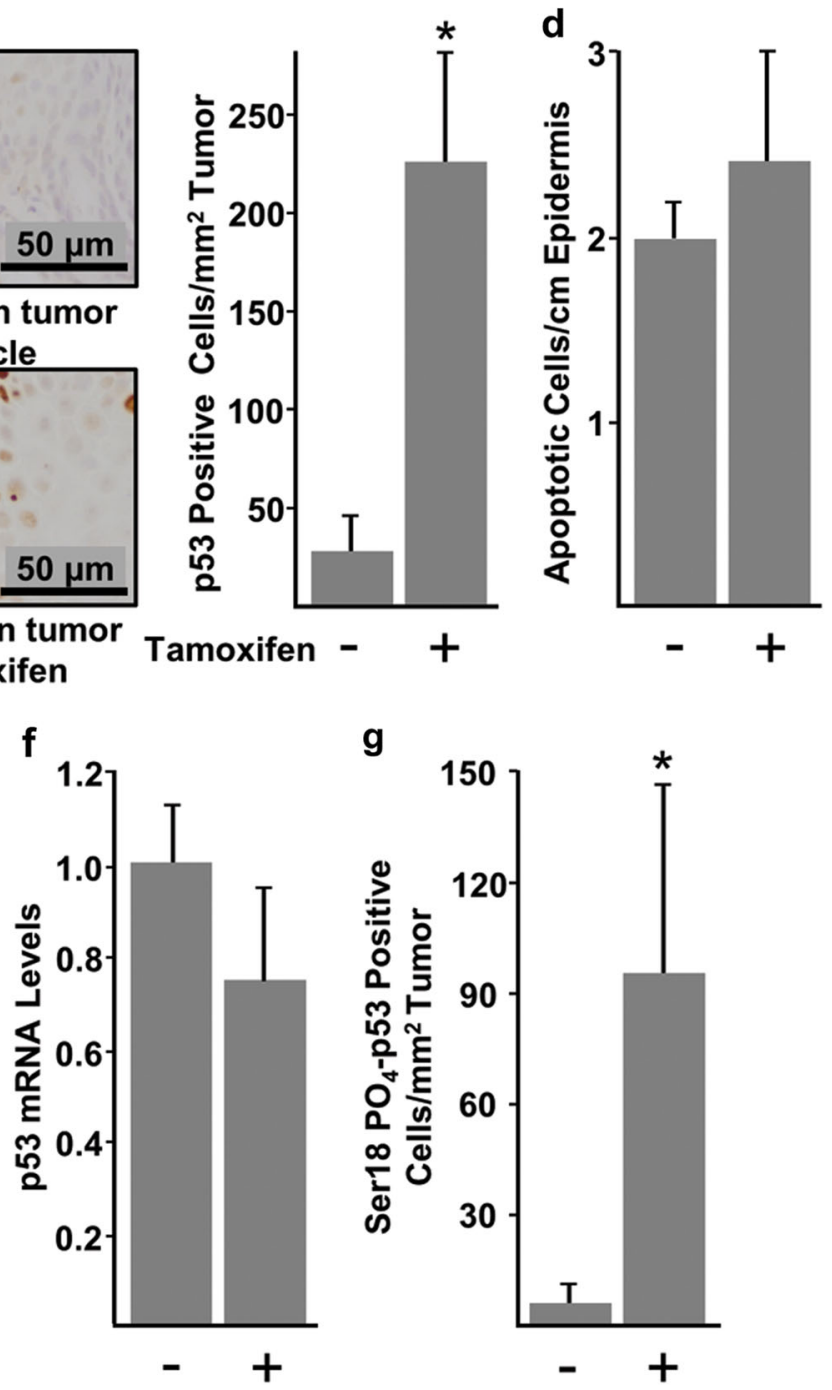

g

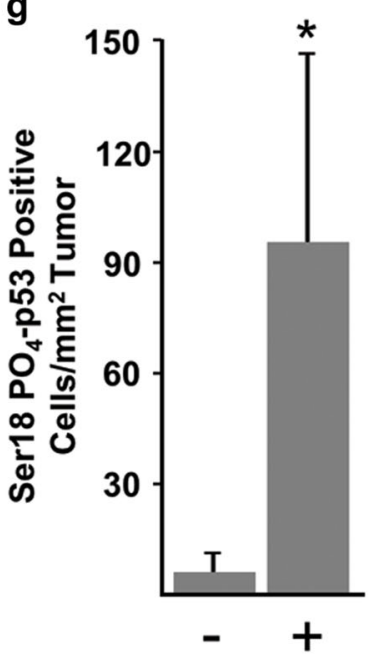

Fig. 3 (See legend on next page.) 
(see figure on previous page)

Fig. 3 Regressing C/EBP $\beta$-deficient tumors display tumor-specific elevations in apoptosis and p53 protein, whereas adjacent C/EBP $\beta$ -

depleted skin is unaffected. Tumor bearing IKO $\beta$ mice were dosed with either vehicle control or tamoxifen at 19 weeks and tumors were collected 2 weeks later. a Deletion of C/EBP $\beta$ was confirmed by IHC staining. b Photograph displaying apoptotic cells (left) and quantification of apoptosis in H\&E-stained tumors (right) (vehicle $n=18$ tumors, tamoxifen $n=10$ tumors). c Photograph displaying p53 IHC staining (left) and quantification of IHC staining for p53 (right) (vehicle $n=15$ tumors, tamoxifen $n=12$ tumors). d Quantification of apoptosis in H\&E-stained adjacent normal epidermis (vehicle $n=5$ mice, tamoxifen $n=8$ mice). e Quantification of IHC staining for p53 in adjacent normal epidermis (vehicle $n=5$ mice, tamoxifen $n=8$ mice). $\mathbf{f}$ Quantification of p53 mRNA from tumors (vehicle $n=3$ tumors, tamoxifen $n=3$ tumor). $\mathbf{g}$ Quantification of IHC staining for p53 phosphorylated on serine 18 (vehicle $n=9$ tumors, tamoxifen $n=11$ tumors). Data are expressed as mean \pm SD. *indicates significantly different from controls $p<0.05$ via the Student's $t$-test

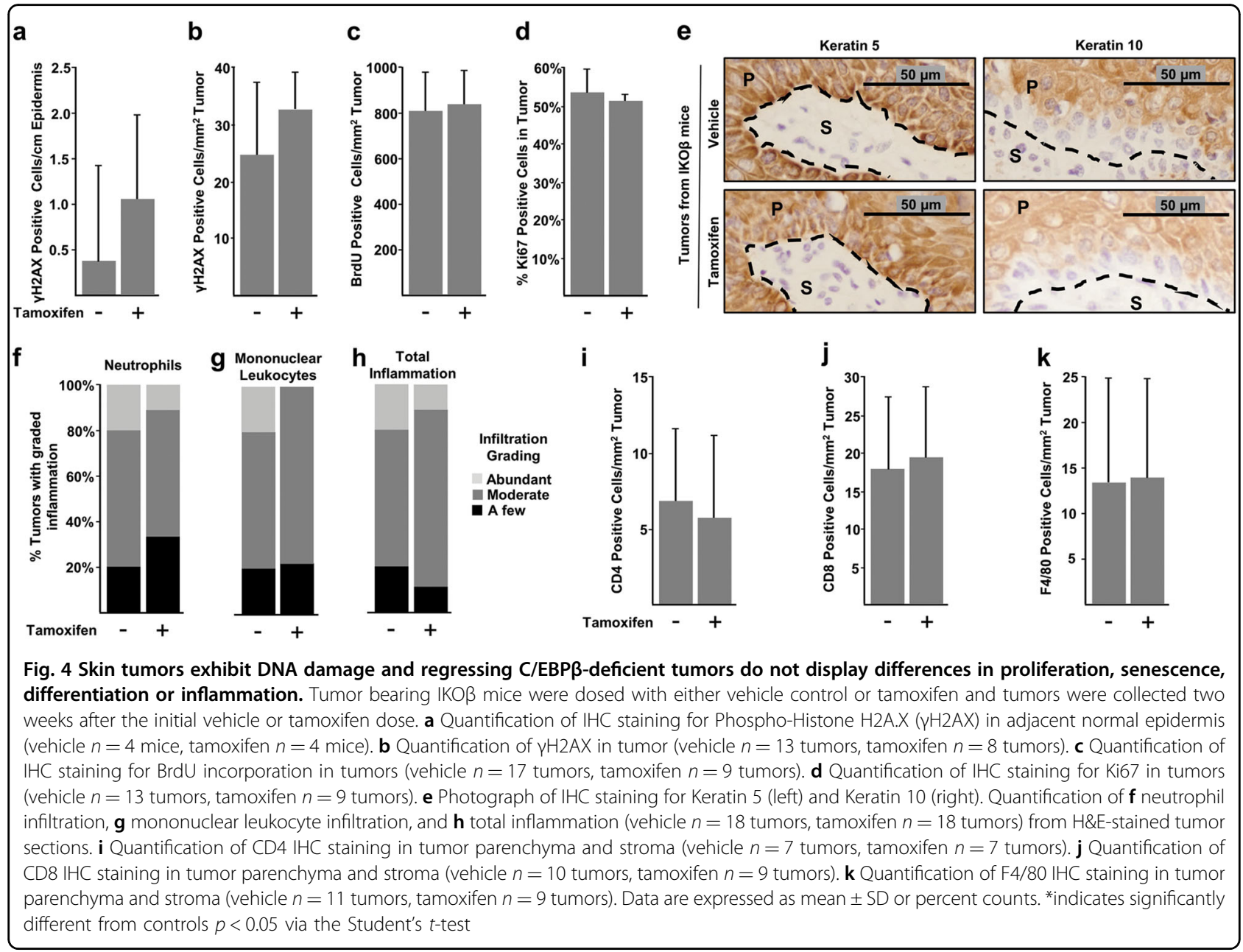

\section{Oncogenic Ras skin tumors are dependent on C/EBP $\beta$ for survival and deletion of $C / E B P \beta$ in these tumors is a synthetic lethal event dependent upon p53}

Cre and DIKO mice were initiated with DMBA, promoted with TPA for 30 weeks, and treated with tamoxifen beginning at week 19 of TPA treatment. No tumor regression was observed in the tamoxifen-treated DIKO and these mice, and similar to the Cre mice, continued to develop additional tumors (Fig. 5e, f). Cre and DIKO mice displayed an increase in tumor volume after tamoxifen treatment (Fig. $5 \mathrm{~g}$ ) and there was no decrease in the percent mice with tumors in either group (Fig. 5f).

As part of this experiment, a small group of Cre, IKO $\beta$, IKOp53, and DIKO mice were also initiated, promoted, and treated with tamoxifen and tumors from these mice were collected for further analysis 2 weeks after the start of tamoxifen treatment. IKO $\beta$ mice displayed tumor regression, whereas Cre, IKOp53, and DIKO mice showed no regression (Fig. 5h) and displayed 


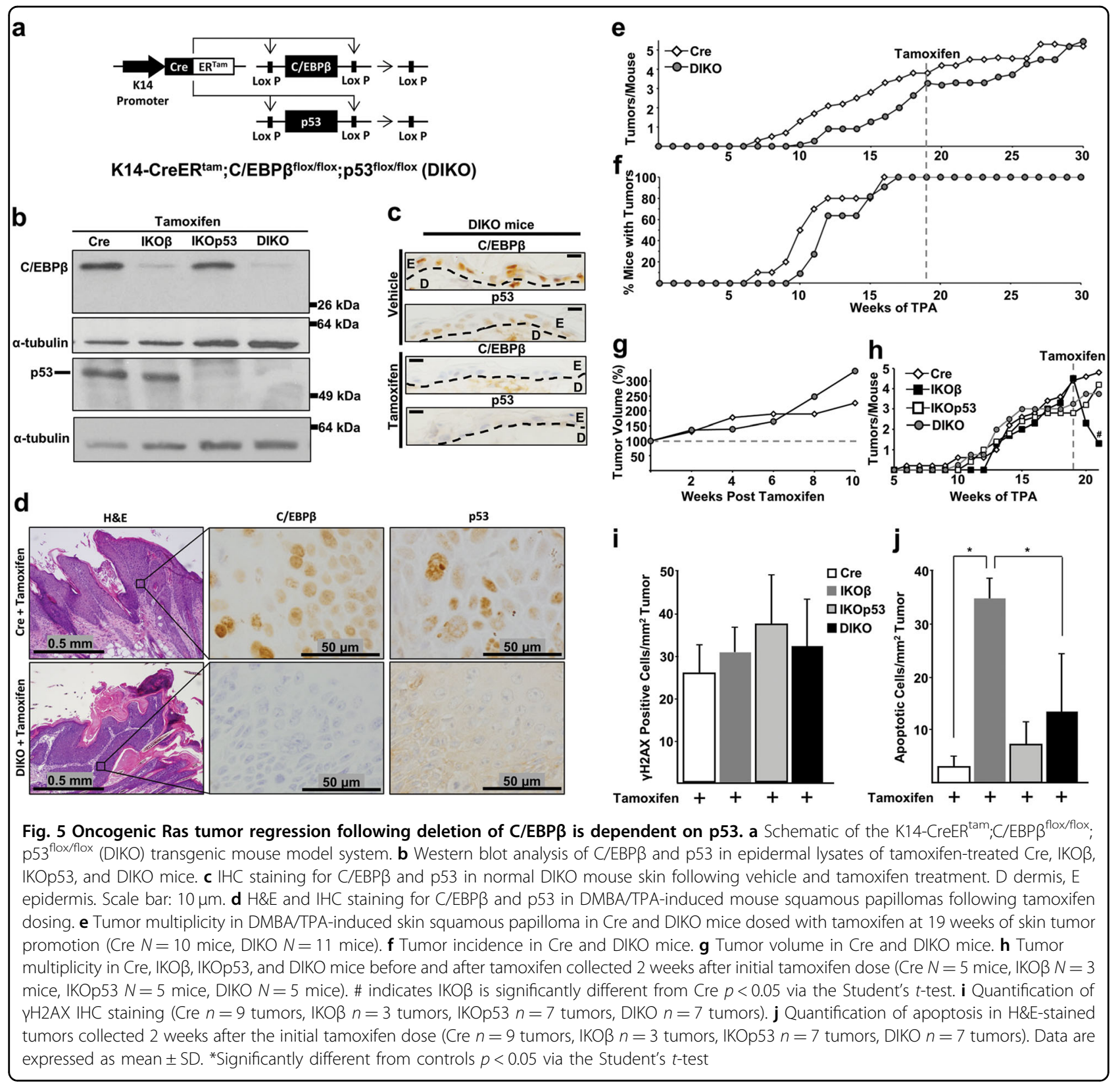

a similar number of tumors/mouse. PFPE sections of the tumors from Cre, IKO $\beta$, IKOp53, and DIKO mice were analyzed for apoptosis and $\gamma \mathrm{H} 2 \mathrm{AX}$ levels. All tumors displayed a tumor stress phenotype involving DNA damage stress as determined by significant of $\gamma \mathrm{H} 2 \mathrm{AX}$-positive cells (Fig. 5i).

Most of the apoptosis observed in the IKO $\beta$ mouse tumors was p53 dependent (Fig. 5j). Thus, co-deletion of $\mathrm{C} / \mathrm{EBP} \beta$ and $\mathrm{p} 53$ in oncogenic Ras tumors demonstrates that p53 is required for tumor regression and elevated apoptosis.
Regressing tumors display enrichment of a type I IFN response, p53, and TNF/death receptor signaling networks

To further understand the mechanism(s) responsible for synthetic lethality, we conducted RNA sequncing (RNAseq) analysis on RNA isolated from three nonregressing tumors (Cre mice) and three regressing tumors (IKO $\beta$ mice) at 2 weeks after the start of tamoxifen treatment. Deletion of $\mathrm{C} / \mathrm{EBP} \beta$ in the tumors had a profound effect on transcriptional responses. We identified a total of 2287 genes (880 upregulated and 1407 downregulated) out of a data set of 18,924 unique genes that 


\section{a}

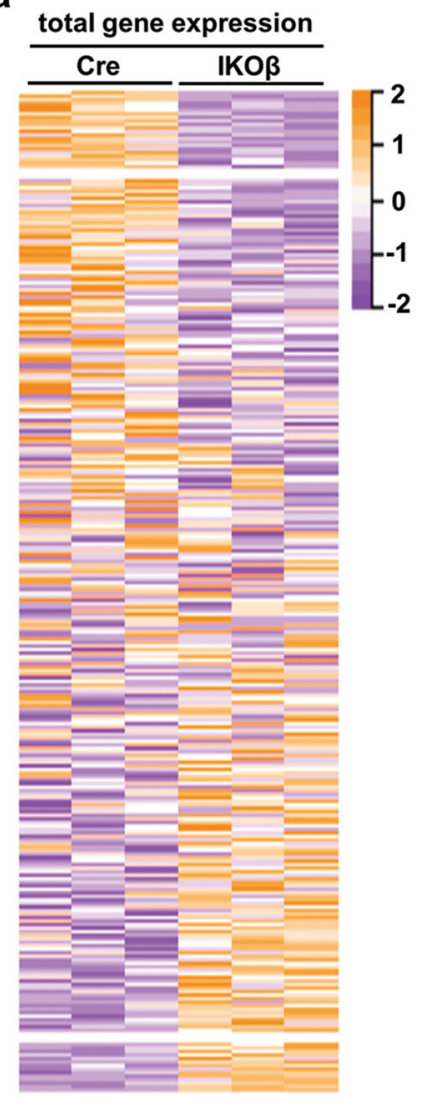

b

HALLMARK_INTERFERON_GAMMA_R. HALLMARK_INTERFERON_ALPHA_RE.. HALLMARK_INFLAMMATORY_RESPO... INTERFERON SIGNALING\%REACTOME... HALLMARK_TNFA_SIGNALING_VIA_NF... CYTOKINE SIGNALING IN IMMUNE SYS. GENE EXPRESSION\%REACTOME DA. INTERFERON ALPHA BETA SIGNALING... HALLMARK_E2F_TARGETS\%MSIGDB... IMMUNE SYSTEM\%REACTOME DATAB... CELL CYCLE\%REACTOME DATABASE... INTERFERON GAMMA SIGNALING\%RE... CELL CYCLE, MITOTIC\%REACTOME\%. CELL CYCLE CHECKPOINT\%REACTO... EGFR1\%OB\%EGFR

G2 M CHECKPOINTS\%REACTOME DA... DNA REPAIR\%REACTOME DATABASE.. SYNTHESIS OF DNA\%REACTOME DA.. EGFR1 \%NETPATH\%EGFR1 S PHASE\%REACTOME DATABASE ID... DNA REPLICATION\%REACTOME DATA. HALLMARK_MYC_TARGETS_V1\%MSIG. HALLMARK_HYPOXIA\%MSIGDB_C2\%H... HALLMARK_GLYCOLYSIS\%MSIGDB_C2... INFECTIOUS DISEASE\%REACTOME DA... HALLMARK_DNA_REPAIR\%MSIGDB_C2... GENERIC TRANSCRIPTION PATHWAY... TRANSCRIPTIONAL REGULATION BY TP53... SIGNALING BY INTERLEUKINS\%REACT... GLUCOCORTICOID RECEPTOR REGULAT... ANTIGEN PROCESSING: UBIQUITINATION... PID_REG_GR_PATHWAY\%MSIGDB_C2\%.. CLASS I MHC MEDIATED ANTIGEN PRO. RIG-I MDA5 MEDIATED INDUCTION OF IFN... UB-SPECIFIC PROCESSING PROTEASES.

HIV INFECTION\%REACTOME\%R-HSA... C-TYPE LECTIN RECEPTORS (CLRS)\%RE... APOPTOSIS SIGNALING PATHWAY\% PAN... HALLMARK_P53 PATHWAY\%MSIGDB_C2... HALLMARK_IL6_JAK_STAT3_SIGNALING...

$$
\begin{array}{cccc}
\hline 0 & 2 & 4 & 6 \\
\text { ment } & \text { Scores }
\end{array}
$$

Fig. 6 Regressing tumors display significant alterations in gene expression and enrichment of type I interferon response, p53, and TNF/ death receptor signaling networks. Total RNA from three Cre and three IKO $\beta$ tumors from different mice were subjected to RNA sequencing. a Heatmap showing total gene expression. b Gene set enrichment analysis with top 40 statistically significant (FDR $<0.1)$, positively enriched pathways displayed

were altered in the regressing tumors compared with the non-regressing tumors $(\mathrm{FDR}<0.1)$ (Fig. 6a and Table S1). Expression patterns in the three regressing tumors were similar and distinct from the expression patterns observed in the three non-regressing tumors (Fig. 6a). GSEA was performed using gene sets from within the Molecular Signature Database (MSigDB). Strikingly, GSEA revealed that the IFN pathway was the most highly enriched pathway in the regressing tumors (Fig. 6b, Table S2). GSEA normalized enrichment scores for the TNF pathway, cell cycle checkpoints/DNA repair, and p53 pathway were also among highest in the regressing tumor $($ FDR $<0.1)$ (Fig. $6 b)$.

\section{Deletion of C/EBP $\beta$ in oncogenic Ras tumors triggers a} subset of ISGs that are unique to the regressing tumors

Among the 2287 significantly altered genes, it is noteworthy that 16 of the top 50 genes were ISGs (Table S1). Further analysis of the MSigDB combined IFN ontologies showed that 48/161 IFN-stimulated genes (ISGs) were significantly altered in the regressing tumor $(\mathrm{FDR}<0.1)$ (Fig. 7a and Table S3). TaqMAN quantittative reversetranscriptase PCR analysis for Ifn $\beta 1$ using RNA isolated from 3 regressing and 3 non-regressing tumors revealed that Ifn $\beta 1$ transcripts were not detectable in non-regressing tumors (40 cycles); however, Ifn $\beta 1$ transcripts were increased $\sim 100-$ fold (average Ct 33 cycles) in the regressing tumors. Unlike the deletion of $C / E B P \beta$ in oncogenic Ras skin tumors, the deletion of C/EBP $\beta$ in mouse epidermis had no effect on apoptosis. To determine whether the 48 ISGs that are significantly altered in the regressing tumor depleted of $C / E B P \beta$ are unique to the regressing tumor, we conducted RNAseq analysis on C/EBP $\beta$-deleted epidermis from three $\mathrm{K} 5 \mathrm{Cre}^{+/ \mathrm{tg}} ; \mathrm{C} / \mathrm{EBP} \beta^{\text {flox/flox }}$ and on epidermis from three K5Cre mice. Surprisingly, many ISGs were also significantly altered in the C/EBP $\beta$-deleted epidermis and, of these, $56 \%$ overlapped with the 48 ISGs significantly altered in the regressing tumor (Fig. 7b). The 21 ISGs whose expression is unique to the regressing tumor are shown in Fig. 7b, and include Irf1, Irf7, and Stat1. 


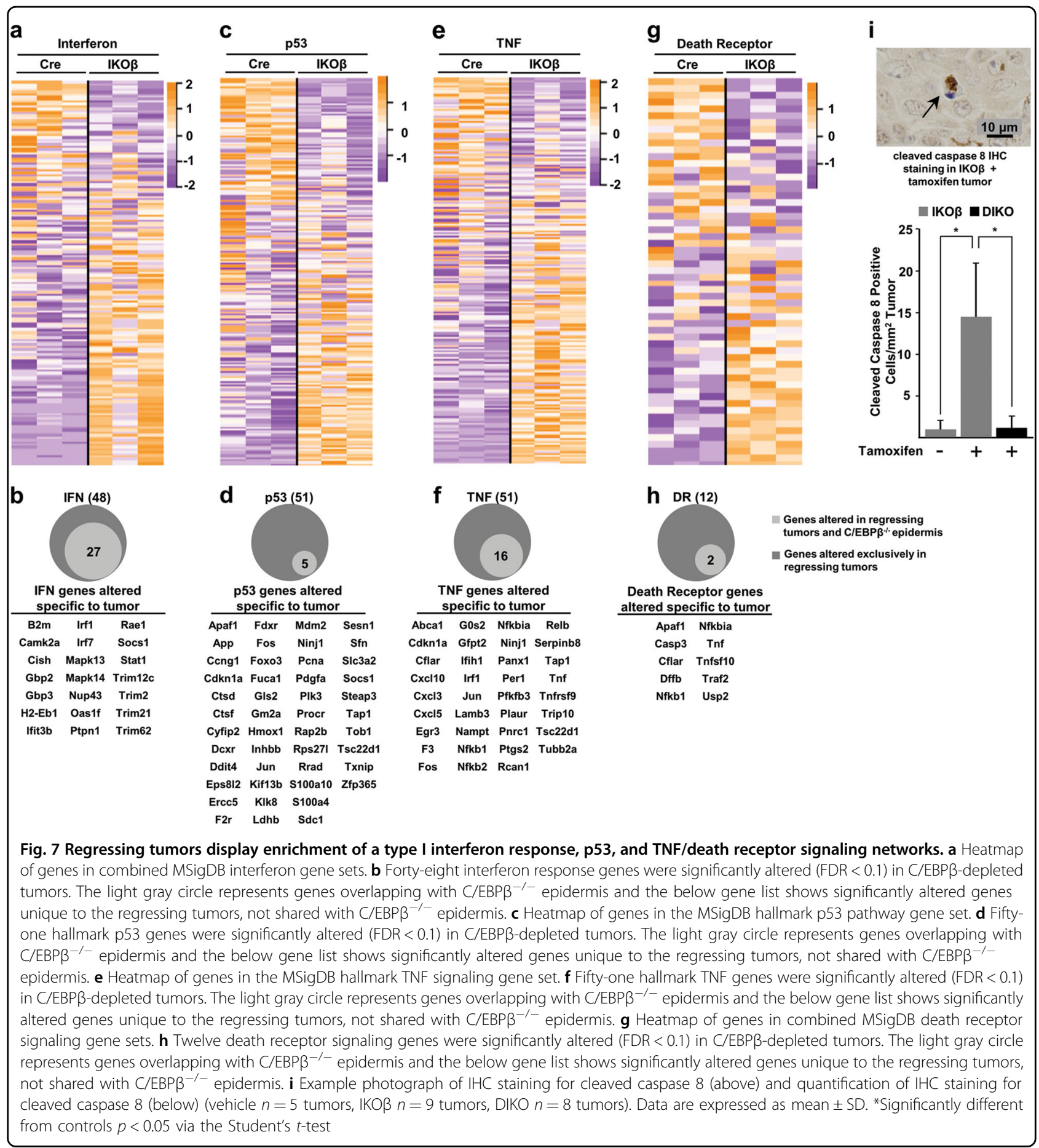

\section{Deletion of C/EBP $\beta$ results in upregulation of $p 53$ target genes involved in the extrinsic apoptotic pathway}

Analysis of MSigDB p53 ontology showed that 51/191 genes associated with the p53 pathway were significantly altered in the regressing tumor $(\mathrm{FDR}<0.1)$ (Fig. 7c, Table S4). Less than $10 \%$ of the significantly altered genes associated with p53 pathway in the regressing tumor are significantly altered in $\mathrm{C} / \mathrm{EBP} \beta$-deleted epidermis further supporting the importance of p53 pathway in tumor regression. The $46 \mathrm{p} 53$ genes whose expression is unique to the regressing tumor and not overlapping with $\mathrm{C} /$ EBP $\beta$-deleted epidermis are shown in Fig. 7d. Strikingly, we did not observe altered expression of p53-dependent genes such as Puma, Bax, and Noxa or other genes involved in the intrinsic apoptotic pathway such as Bim, $B a d, B i d$, and Bmf in the regressing tumors. We did 
observe that Apaf1 was significantly and selectively upregulated in regressing tumors (FDR $<0.1$ ).

\section{TNF/death receptor pathways are activated in the regressing tumors}

Similar to GSEA, Ingenuity Pathway Analysis (IPA) of regressing and non-regressing tumors also showed that the TNF, IFN, and p53/apoptosis pathways as top canonical pathways altered in the regressing tumors; however, IPA identified the death receptor pathway (22/93 genes) as the number one canonical pathway $(z$-score $=3.8)$ associated with regressing tumors. Analysis of the gene sets for the TNF and death receptor pathways revealed that 51/195 genes (Fig. 7e and Table S5) and 12/58 genes (Fig. $7 \mathrm{~g}$ and Table S6), respectively, were significantly altered in the regressing tumors $(F D R<0.1)$. Of the genes within the TNF and death receptor gene sets that were significantly altered in the regressing tumors, only 31\% (16/51) TNF and $17 \%(2 / 12)$ death receptor genes were significantly altered in C/EBP $\beta$-deleted epidermis (Fig. $7 \mathrm{f} / \mathrm{h}$ ). The 35 TNF genes and the 10 death receptor genes whose expression is unique to the regressing tumor and not overlapping with $\mathrm{C} / \mathrm{EBP} \beta$-deleted epidermis are shown in Fig. $7 \mathrm{f} / \mathrm{h}$. Some of these key genes in TNF and death receptor pathways include Tnf, Cflar (cFLIP), Traf2, Tnfrsf9, and Tnfsf10 (TRAIL). Caspase 8 activation (cleaved caspase 8 ) is a key part of the extrinsic apoptotic pathway and caspase 8 is primarily activated by death receptor and TNFR signaling. To provide evidence that the TNF/death receptor pathways are activated in the regressing tumor, we conducted IHC analysis for cleaved caspase 8 in regressing and non-regressing tumors. IHC analysis revealed regressing tumors displayed a remarkable 14-fold increase in cleaved caspase 8 when compared with nonregressing tumors (Fig. 7i). Moreover, this increase in cleaved caspase 8 was not observed in DIKO tumors.

\section{Discussion}

Previous studies showed that mice lacking C/EBP $\beta$ in their epidermis are highly resistant to the development of skin tumors by chemical carcinogens that produce mutations in $\mathrm{Ha}-\mathrm{Ras}^{18}$. To determine whether $\mathrm{C} / \mathrm{EBP} \beta$ is essential for the survival of oncogenic Ha-Ras tumors, we developed a mouse model where C/EBP $\beta$ could be conditionally and temporally deleted in oncogenic Ras tumors. We show that deletion of $\mathrm{C} / \mathrm{EBP} \beta$ in oncogenic Ras tumors resulted in rapid tumor regression that was accompanied by elevated levels of apoptosis and p53. Importantly, this increase in apoptosis and p53 was tumor specific, as neither apoptosis nor p53 levels were increased in $\mathrm{C} / \mathrm{EBP} \beta$-depleted epidermis adjacent to the tumor. Our results demonstrate that the deletion of $C / E B P \beta$ in oncogenic Ras skin tumors is a synthetic lethal event dependent upon p53.
Large-scale synthetic lethal screens using cultured cells and RNA interference have been conducted to identify genes that are required for survival of oncogenic Ras containing cells ${ }^{39}$. In general, these screens have not yielded the positive results initially anticipated and it has been suggested that this is, in part, due to the in vitro screening methods utilized, which do not replicate the selective pressures experienced by tumor cells in vivo ${ }^{39}$. Although our in vivo studies have overcome this in vitro issue, most human tumors display Ki-Ras mutations ${ }^{1}$ and our in vivo mouse skin model exclusively involves Ha-Ras ${ }^{\mathrm{Q} 61 \mathrm{~L}}$ mutations. Future studies will be required to determine if the synthetic lethal effect of $\mathrm{C} / \mathrm{EBP} \beta$ deletion in vivo in skin tumors is applicable to other mouse model systems/tumor types and forms of oncogenic Ras ( $\mathrm{Ha}, \mathrm{Ki}, \mathrm{N})$.

Previous studies showed C/EBP $\beta$ knockout mice treated with DNA-damaging agents display elevated levels of p53 and p53-mediated apoptosis in their epidermis compared with similarly treated wild-type mice $^{22,23}$. These findings indicate that $C / E B P \beta$ functions to repress p53 levels/activity and increase cell survival in response to exogenously induced DNA damage. In the current study, we observed elevated levels of DNA damage (based on increased levels of $\gamma \mathrm{H} 2 \mathrm{AX})$ in both regressing and non-regressing oncogenic Ras skin tumors ${ }^{58}$. DNA damage in tumors is known to be induced by oncogenic stress and can occur through stalled replication forks and reactive oxygen species $^{38,59}$. Therefore, we reasoned that the deletion of $\mathrm{C} / \mathrm{EBP} \beta$ may sensitize oncogenic Ras skin tumor cells to the endogenous levels of DNA damage through derepression of p53 activity. Increased endogenous DNA damage stress is a tumorigenic stress phenotype that tumors are forced to deal with, in order to survive ${ }^{38,59}$ and, in doing so, the tumor may become over reliant on genes such as $C / E B P \beta$ to suppress apoptosis downstream of DNA damage. Luo et al. ${ }^{38}$ suggested selective tumor cell killing could be accomplished by stress sensitization or stress overload. Stress sensitization involves blocking a pathway(s) the tumor has evolved to depend on to handle the increased tumorigenic stress, whereas stress overload involves causing additional stress to the tumor resulting in an overload of the already stretched capacity of the tumor to handle this cellular stress. Our results are consistent with the notion that the deletion of $C / E B P \beta$ removes a critical stress survival pathway in the tumor and this sensitizes tumor cells to oncogenic stress and tumorigenic stress-induced endogenous DNA damage to stimulate synthetic lethal apoptotic cell death and tumor regression.

Our cleaved caspase 8 results are consistent with the idea that the extrinsic apoptosis pathway involving TNF/ TRAIL (TNF-related apoptosis-inducing ligand)/death receptor are activated in the regressing tumor and that 
p53 is essential for this activation in the C/EBP $\beta$-deleted skin tumors. However, caspase 8 has been reported to be activated by caspase 3 via the intrinsic apoptosis pathways as part of a feedback loop ${ }^{60}$, so we cannot rule out the intrinsic pathways of apoptosis. When comparing regressing vs non-regressing tumors we observed no differences in the expression of key genes involved in p53dependent intrinsic apoptosis such as Noxa and Puma ${ }^{61}$ or genes involved in Endoplasmic Reticulum (ER) stressinduced apoptosis such as Bim, Perk, Ire1, and Atf6 ${ }^{62,63}$. Although no differences in Puma, Noxa, and Bim mRNA levels were detected, it is conceivable that altered posttranslational regulation in the regressing tumors results in increased protein levels of these key genes. It is also possible that the existing levels of Puma, Noxa, and Bim in the regressing tumor are sufficient to cooperate/synergize with the observed upregulation of the extrinsic apoptosis pathway to regulate cell death. Although DNA damage can induce apoptosis through a p53-dependent intrinsic pathway independent of Fas or the death receptor adapter FADD $^{64}$, it has also been reported that TRAIL can mediate p53-dependent apoptosis through the death receptor DR5 in response to DNA damage ${ }^{65,66}$. Our unbiased approaches involving RNAseq analysis strongly point to activation of extrinsic apoptosis pathways exclusively in regressing tumors. Based on our phenotypic results and key genes significantly altered $(\mathrm{FDR}<0.1)$ in the regressing tumors we have developed a working model (Fig. 8), whereby we link oncogenic Ras, DNA damage, type I IFN response, TNF, TRAIL, death receptors, and p53 pathway to the apoptotic response and tumor regression observed in $C / E B P \beta$-depleted tumors. In this model, we propose the deletion of C/EBP $\beta$ de-represses p53 and sensitizes tumor cells to the DNA damage induced by oncogenic Ras/tumor stress, and this coupled with an induced type- 1 IFN response further activates the de-repressed p53 producing a positive feedback loop, which initiates extrinsic apoptosis signaling and tumor regression through the TNFR and death receptor pathways ${ }^{67,68}$.

IRF7 is activated by pattern-recognition receptors that recognize nucleic acids ${ }^{69}$ and is significantly increased in the regressing tumor. IRF7 is a potent transcriptional regulator of many ISGs including of IFN $\alpha / \beta^{69}$, and in our model, we propose this results in a continuous stimulation of type-1 IFN response. The expression of numerous key significantly altered ISGs in our GSEA data sets are also regulated by $\mathrm{p} 53$ and several ISGs positively regulate $\mathrm{p} 53^{70}$. For example, several of the upregulated ISGs in the regressing tumors such as Irf9, Stat1, Stat2, Tnf, TRAIL,

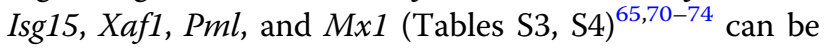
regulated by IFN or p53. IRF9, STAT1, and STAT2 form the canonical heterotrimeric ISG factor 3 protein complex, which regulates the transcription of a multitude of ISGs including $\mathrm{p} 53^{71,75}$. Some of the above mentioned

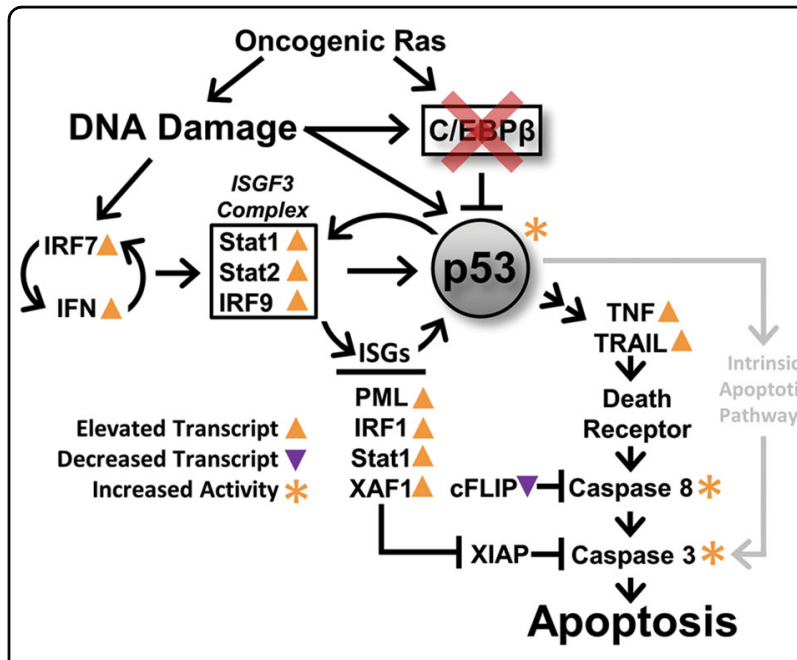

Fig. 8 Positive Feedback Working Model. Model depicting the link between oncogenic Ras, DNA damage, type I interferon response, TNF, death receptors, and the p53 pathway to the apoptotic response and tumor regression observed in C/EBP $\beta$-depleted tumors. Arrows and asterixes represent statistically significant up- (orange) or downregulated (purple) transcripts (FDR $<0.1)$ or proteins $(p<0.05)$ in the $C / E B P \beta$-depleted regressing tumors

ISGs, including STAT1, PML, IRF1, ISG15, and XAF1 can positively regulate p53 protein activity/stability ${ }^{71-73,76,77}$. This results in a positive feedback loop involving ISGs and de-repressed p53 initiates apoptosis through the TNFR, TRAIL, and death receptor pathways ${ }^{67,68}$. The ISG Xaf1 is significantly upregulated in the regressing tumors, and XAF1 and TRAIL are major mediators of the extrinsic cell death pathway where XAF1 significantly increases the cellular sensitivity to the pro-apoptotic actions of TRAIL $^{78}$. We also observed a significant downregulation of Cflar (cFLIP) a master anti-apoptotic regulator of TNFand TRAIL-induced apoptosis ${ }^{68}$.

Although p53 is most appreciated for its role in DNA damage and oncogenic stress, it is now evident that p53 has important roles in the immune system, including roles in innate immunity where it enhances type-1 IFN activity $^{70}$. Our results indicate that loss of C/EBP $\beta$ in oncogenic Ras tumor cells unleashes a hyperactive type-1 IFN and reveals a novel interface between p53, innate immune response, and death receptor pathways that function together produce tumor regression. Targeting of $C / E B P \beta$ could hold promise as future potential cancer therapy.

\section{Acknowledgements}

This work was supported by a grants from the National Cancer Institute (CA046637) awarded to R.C.S. and from the National Institute of Environmental Health Sciences (ES024471) awarded to R.C.S. Z.J.M. was supported by a training grant from the National Institute of Environmental Health Sciences (ES007046). Additional support for this research was provided by the National Institute of Environmental Health Sciences(P30ES025128) through the NC State University Center for Human Health and the Environment (CHHE), and its Comparative Pathology, Bioinformatics, and Genomics Cores. 


\section{Author details}

${ }^{1}$ Toxicology Program, Raleigh, NC, USA. ${ }^{2}$ Center of Human Health and the Environment, Raleigh, NC, USA. ${ }^{3}$ Department of Biological Sciences, Raleigh, NC, USA. ${ }^{4}$ Bioinformatics Research Center, Raleigh, NC, USA. ${ }^{5}$ Department of Population Health and Pathobiology, North Carolina State University, Raleigh, NC, USA

\section{Author contributions}

Z.J.M., J.R.H., and R.C.S. developed the concept and designed experiments. Z.J. M., J.R.H., and H.W.T. performed experiments. Z.J.M., J.R.H., D.D.J., J.S.H., D.A.T., and R.C.S. analyzed data. Z.J.M., J.R.H., and R.C.S. wrote the manuscript.

\section{Conflict of interest}

The authors declare that they have no conflict of interest.

\section{Publisher's note}

Springer Nature remains neutral with regard to jurisdictional claims in published maps and institutional affiliations.

Supplementary Information accompanies this paper at (https://doi.org/ 10.1038/s41419-018-1103-y).

Received: 20 August 2018 Accepted: 22 August 2018

Published online: 15 October 2018

\section{References}

1. Stephen, A. G., Esposito, D., Bagni, R. K. \& McCormick, F. Dragging ras back in the ring. Cancer Cell. 25, 272-281 (2014).

2. Pylayeva-Gupta, Y., Grabocka, E. \& Bar-Sagi, D. RAS oncogenes: weaving a tumorigenic web. Nat. Rev. Cancer 11, 761-774 (2011).

3. Malumbres, M. \& Barbacid, M. RAS oncogenes: the first 30 years. Nat. Rev. Cancer 3, 459-465 (2003).

4. Sun, H., Tonks, N. K. \& Bar-Sagi, D. Inhibition of Ras-induced DNA synthesis by expression of the phosphatase MKP-1. Science 266, 285-288 (1994).

5. Jimenez, $C$. et al. Identification and characterization of a new oncogene derived from the regulatory subunit of phosphoinositide 3-kinase. EMBO J. 17, 743-753 (1998).

6. Khosravi-Far, R. et al. Oncogenic Ras activation of Raf/mitogen-activated protein kinase-independent pathways is sufficient to cause tumorigenic transformation. Mol. Cell. Biol. 16, 3923-3933 (1996).

7. Urano, T., Emkey, R. \& Feig, L. A. Ral-GTPases mediate a distinct downstream signaling pathway from Ras that facilitates cellular transformation. EMBO J. 15 810-816 (1996).

8. White, M. A., Vale, T., Camonis, J. H., Schaefer, E. \& Wigler, M. H. A role for the Ral guanine nucleotide dissociation stimulator in mediating Ras-induced transformation. J. Biol. Chem. 271, 16439-16442 (1996).

9. Cowley, S., Paterson, H., Kemp, P. \& Marshall, C. J. Activation of MAP kinase kinase is necessary and sufficient for PC12 differentiation and for transformation of NIH 3 T3 cells. Cell 77, 841-852 (1994).

10. Cox, A. D. \& Der, C. J. The dark side of Ras: regulation of apoptosis. Oncogene 22, 8999-9006 (2003).

11. Ramji, D. P. \& Foka, P. CCAAT/enhancer-binding proteins: structure, function and regulation. Biochem. J. 365(Pt 3), 561-575 (2002).

12. Tsukada, J., Yoshida, Y., Kominato, Y., \& Auron, P. E. The CCAAT/enhancer (C/EBP) family of basic-leucine zipper (bZIP) transcription factors is a multifaceted highly-regulated system for gene regulation. Cytokine 54: 6-19 (2011).

13. House, J. S., Zhu, S., Ranjan, R., Linder, K. \& Smart, R. C. C/EBPalpha and C/EBPbeta are required for Sebocyte differentiation and stratified squamous differentiation in adult mouse skin. PLOS ONE 5, e9837 (2010).

14. Matsusaka, T. et al. Transcription factors NF-IL6 and NF-kB synergistically activate transcription of the inflammatory cytokine interleukin 6 and interleukin 8 . PNAS 90, 10193-10197 (1993).

15. Akagi, T. et al. Impaired response to GM-CSF and G-CSF, and enhanced apoptosis in C/EBPbeta-deficient hematopoietic cells. Blood 111, 2999-3004 (2008).

16. Armstrong, D. A., Phelps, L. N. \& Vincenti, M. P. CCAAT enhancer binding protein-beta regulates matrix metalloproteinase-1 expression in interleukin- 1beta-stimulated A549 lung carcinoma cells. Mol. Cancer Res. 7, 1517-1524 (2009).

17. Nakajima, T. et al. Phosphorylaton at threonine-235 by a ras dependent mitogen-activated protein kinase cascade is essential for transcription factor NF-IL6. Proc. Natl Acad. Sci. USA 90, 2207-2211 (1993).

18. Zhu, S., Yoon, K., Sterneck, E., Johnson, P. F. \& Smart, R. C. CCAAT/enhancer binding protein-beta is a mediator of keratinocyte survival and skin tumorigenesis involving oncogenic Ras signaling. Proc. Natl Acad. Sci. USA 99, 207-212 (2002)

19. Buck, M., Poli, V., Geer, Pvd, Chojkier, M. \& Hunter, T. Phosphorylation of rat serine 105 or mouse threonine 217 in C/EBPbeta is required for hepatocyte proliferation induced by TGFalpha. Mol. Cell 4, 1087-1092 (1999).

20. Cho, I. J., Woo, N. R. \& Kim, S. G. The identification of C/EBPbeta as a transcription factor necessary for the induction of MAPK phosphatase-1 by toll-like receptor-4 ligand. Arch. Biochem. Biophys. 479, 88-96 (2008).

21. Lu, Y. C. et al. Differential role for C-Rel and C/EBPbeta/delta in TLR-mediated induction of proinflammatory cytokines. J. Immunol. 182, 7212-7221 (2009)

22. Ewing, S. J., Zhu, S., Zhu, F., House, J. S. \& Smart, R. C. C/EBPbeta represses p53 to promote cell survival downstream of DNA damage independent of oncogenic Ras and p19(Arf). Cell Death Differ. 15, 1734-1744 (2008).

23. Yoon, K., Zhu, S., Ewing, S. J. \& Smart, R. C. Decreased survival of C/EBP beta-deficient keratinocytes is due to aberrant regulation of p53 levels and function. Oncogene 26, 360-367 (2007).

24. Buck, M., Poli, V., Hunter, T. \& Chojkier, M. C/EBPbeta phosphorylation by RSK creates a functional XEXD caspase inhibitory box critical for cell survival. Mol. Cell 8, 807-816 (2001).

25. Wessells, J., Yakar, S. \& Johnson, P. F. Critical prosurvival roles for C/EBP beta and insulin-like growth factor I in macrophage tumor cells. Mol. Cell. Biol. 24, 3238-3250 (2004).

26. Aguilar-Morante, D., Cortes-Canteli, M., Sanz-Sancristobal, M. A., Santos, A., \& Perez-Castillo, A. Decreased CCAAT/enhancer binding protein beta expression inhibits the growth of glioblastoma cells. Neuroscience 176: 110-119 (2011).

27. Anastasov, N., et al. C/EBPbeta expression in ALK-positive anaplastic large cell lymphomas is required for cell proliferation and is induced by the STAT3 signaling pathway. Haematologica 95: 760-767 (2010).

28. Bundy, L. M. \& Sealy, L. CCAAT/enhancer binding protein beta (C/EBPbeta)-2 transforms normal mammary epithelial cells and induces epithelial to mesenchymal transition in culture. Oncogene 22, 869-883 (2003).

29. Duprez, E. A new role for C/EBPbeta in acute promyelocytic leukemia Cell Cycle 3, 389-390 (2004).

30. Homma, J. et al. Increased expression of CCAAT/enhancer binding protein beta correlates with prognosis in glioma patients. Oncol. Rep. 15, 595-601 (2006).

31. Kim, M. H., Minton, A. Z. \& Agrawal, V. C/EBPbeta regulates metastatic gene expression and confers TNF-alpha resistance to prostate cancer cells. Prostate 69, 1435-1447 (2009).

32. Li, W. et al. A gene expression signature for relapse of primary wilms tumors. Cancer Res. 65, 2592-2601 (2005).

33. Pal, $\mathrm{R}$. et al. C/EBPbeta regulates transcription factors critical for proliferation and survival of multiple myeloma cells. Blood 114, 3890-3898 (2009).

34. Rask, K. et al. Increased expression of the transcription factors CCAAT-rnhance binding brotein-beta and C/EBP-zeta correlate with invasiveness of human colorectal cancer. Int. J. Cancer 86, 337-343 (2000).

35. Sundfeldt, $\mathrm{K}$. et al. The expression of CCAAT/enhancer binding protein in human ovary in vivo: specific increase in C/EBPbeta dering epithelial tumour progression. Br. J. Cancer 79, 1240-1248 (1999).

36. Piva, R. et al. Functional validation of the anaplastic lymphoma kinase signature identifies CEBPB and BCL2A1 as critical target genes. J. Clin. Invest. 116 3171-3182 (2006).

37. Hanahan, D. \& Weinberg, R. A. Hallmarks of cancer: the next generation. Cell 144, 646-674 (2011)

38. Luo, J., Solimini, N. L. \& Elledge, S. J. Principles of cancer therapy: oncogene and non-oncogene addiction. Cell 136, 823-837 (2009).

39. Downward, J. RAS synthetic lethal screens revisited: still seeking the elusive prize?. Clin. Cancer Res. 21, 1802-1809 (2015).

40. Kaelin, W. G. Jr. The concept of synthetic lethality in the context of anticancer therapy. Nat. Rev. Cancer 5, 689-698 (2005).

41. O'Neil, N. J., Bailey, M. L. \& Hieter, P. Synthetic lethality and cancer. Nat. Rev. Genet., 18: 613-623 (2017). 
42. Weinstein, I. B. et al. Disorders in cell circuitry associated with multistage carcinogenesis: exploitable targets for cancer prevention and therapy. Clin. Cancer Res. 3(12 Pt 2), 2696-2702 (1997).

43. Vasioukhin, V., Degenstein, L., Wise, B. \& Fuchs, E. The magical touch: genome targeting in epidermal stem cells induced by tamoxifen application to mouse skin. Proc. Natl Acad. Sci. USA 96, 8551-8556 (1999).

44. Sterneck, E., Zhu, S., Ramirez, A., Jorcano, J. L. \& Smart, R. C. Conditional ablation of C/EBP beta demonstrates its keratinocyte-specific requirement for cell survival and mouse skin tumorigenesis. Oncogene 25, 1272-1276 (2006).

45. Jonkers, J. et al. Synergistic tumor suppressor activity of BRCA2 and p53 in a conditional mouse model for breast cancer. Nat. Genet. 29, 418-425 (2001).

46. Schneider, C. A., Rasband, W. S. \& Eliceiri, K. W. NIH Image to ImageJ: 25 years of image analysis. Nat. Methods 9, 671-675 (2012).

47. Kugelberg, E. et al. Establishment of a superficial skin infection model in mice by using Staphylococcus aureus and Streptococcus pyogenes. Antimicrob. Agents Chemother. 49, 3435-3441 (2005)

48. Dobin, A. et al. STAR: ultrafast universal RNA-seq aligner. Bioinformatics 29, 15-21 (2013).

49. Love, M. I., Huber, W. \& Anders, S. Moderated estimation of fold change and dispersion for RNA-seq data with DESeq2. Genome Biol. 15, 550 (2014).

50. Benjamini, Y. and Hochberg, Y. Controlling the false discovery rate: a practical and powerful approach to multiple testing. J. R. Stat. Soc. Ser. B (Methodol.). 57 289-300 (1995).

51. Subramanian, A. et al. Gene set enrichment analysis: a knowledge-based approach for interpreting genome-wide expression profiles. Proc. Natl Acad. Sci. USA 102, 15545-15550 (2005).

52. Mootha, V. K. et al. PGC-1alpha-responsive genes involved in oxidative phosphorylation are coordinately downregulated in human diabetes. Nat. Genet. 34, 267-273 (2003).

53. Abel, E. L., Angel, J. M., Kiguchi, K. \& DiGiovanni, J. Multi-stage chemical carcinogenesis in mouse skin: fundamentals and applications. Nat. Protoc. 4, 1350-1362 (2009).

54. Quintanilla, M., Brown, K., Ramsden, M. \& Balmain, A. Carcinogen-specific mutation and amplification of Ha-ras during mouse skin carcinogenesis. Nature 322, 78-80 (1986).

55. Banin, S. et al. Enhanced phosphorylation of p53 by ATM in response to DNA damage. Science 281, 1674-1677 (1998).

56. Canman, C. E. et al. Activation of the ATM kinase by ionizing radiation and phosphorylation of p53. Science 281, 1677-1679 (1998).

57. Appella, E. \& Anderson, C. W. Post-translational modifications and activation of p53 by genotoxic stresses. Eur. J. Biochem. 268, 2764-2772 (2001).

58. Kuo, L. J. \& Yang, L. X. Gamma-H2AX - a novel biomarker for DNA doublestrand breaks. In Vivo 22, 305-309 (2008).

59. Gaillard, H., Garcia-Muse, T. \& Aguilera, A. Replication stress and cancer. Nat. Rev. Cancer 15, 276-289 (2015).
60. Ferreira, K. S. et al. Caspase-3 feeds back on caspase-8, Bid and XIAP in type I Fas signaling in primary mouse hepatocytes. Apoptosis 17, 503-515 (2012).

61. Villunger, A. et al. p53- and drug-induced apoptotic responses mediated by BH3-only proteins puma and noxa. Science 302, 1036-1038 (2003).

62. Puthalakath, $\mathrm{H}$. et al. ER stress triggers apoptosis by activating $\mathrm{BH} 3$-only protein Bim. Cell 129, 1337-1349 (2007).

63. Sano, R. \& Reed, J. C. ER stress-induced cell death mechanisms. Biochim Biophys. Acta 1833, 3460-3470 (2013).

64. Newton, K. \& Strasser, A. lonizing radiation and chemotherapeutic drugs induce apoptosis in lymphocytes in the absence of Fas or FADD/ MORT1 signaling. Implications for cancer therapy. J. Exp. Med. 191, 195-200 (2000).

65. Kuribayashi, $K$. et al. TNFSF10 (TRAIL), a p53 target gene that mediates p53-dependent cell death. Cancer Biol. Ther. 7, 2034-2038 (2008).

66. Finnberg, N. et al. DR5 knockout mice are compromised in radiation-induced apoptosis. Mol. Cell Biol. 25, 2000-2013 (2005).

67. Wang, S. \& El-Deiry, W. S. TRAIL and apoptosis induction by TNF-family death receptors. Oncogene 22, 8628-8633 (2003).

68. Safa, A. R. c-FLIP, a master anti-apoptotic regulator. Exp. Oncol. 34, 176-184 (2012).

69. Honda, K. \& Taniguchi, T. IRFs: master regulators of signalling by Toll-like receptors and cytosolic pattern-recognition receptors. Nat. Rev. Immunol. 6 , 644-658 (2006).

70. Miciak, J. \& Bunz, F. Long story short: p53 mediates innate immunity. Biochim Biophys. Acta 1865, 220-227 (2016).

71. Munoz-Fontela, C., Mandinova, A., Aaronson, S. A. \& Lee, S. W. Emerging roles of p53 and other tumour-suppressor genes in immune regulation. Nat. Rev. Immunol. 16, 741-750 (2016).

72. Zhang, F. \& Sriram, S. Identification and characterization of the interferon-betamediated p53 signal pathway in human peripheral blood mononuclear cells. Immunology 128(1 Suppl), e905-e918 (2009).

73. Zou, B. et al. XIAP-associated factor 1 (XAF1), a novel target of p53, enhances p53-mediated apoptosis via post-translational modification. Mol. Carcinog. 51 422-432 (2012).

74. Munoz-Fontela, C. et al. Transcriptional role of p53 in interferon-mediated antiviral immunity. J. Exp. Med. 205, 1929-1938 (2008).

75. Ivashkiv, L. B. \& Donlin, L. T. Regulation of type I interferon responses. Nat. Rev. Immunol. 14, 36-49 (2014).

76. Townsend, P. A. et al. STAT-1 interacts with p53 to enhance DNA damageinduced apoptosis. J. Biol. Chem. 279, 5811-5820 (2004).

77. Pampin, M., Simonin, Y., Blondel, B., Percherancier, Y. \& Chelbi-Alix, M. K Cross talk between PML and p53 during poliovirus infection: implications for antiviral defense. J. Virol. 80, 8582-8592 (2006).

78. Micali, O. C. et al. Silencing of the XAF1 gene by promoter hypermethylation in cancer cells and reactivation to TRAIL-sensitization by IFN-beta. BMC Cancer 7, 52 (2007). 